\title{
Development of a stagnation streamline model for thermochemical nonequilibrium flow
}

Cite as: Phys. Fluids 32, 046102 (2020); https://doi.org/10.1063/5.0003247

Submitted: 01 February 2020 . Accepted: 13 March 2020 . Published Online: 01 April 2020

Qizhen Hong (洪启臻) (D, Xiaoyong Wang (王小永) (D), Yuan Hu (胡远) (D), and Quanhua Sun (孙泉华) (D)
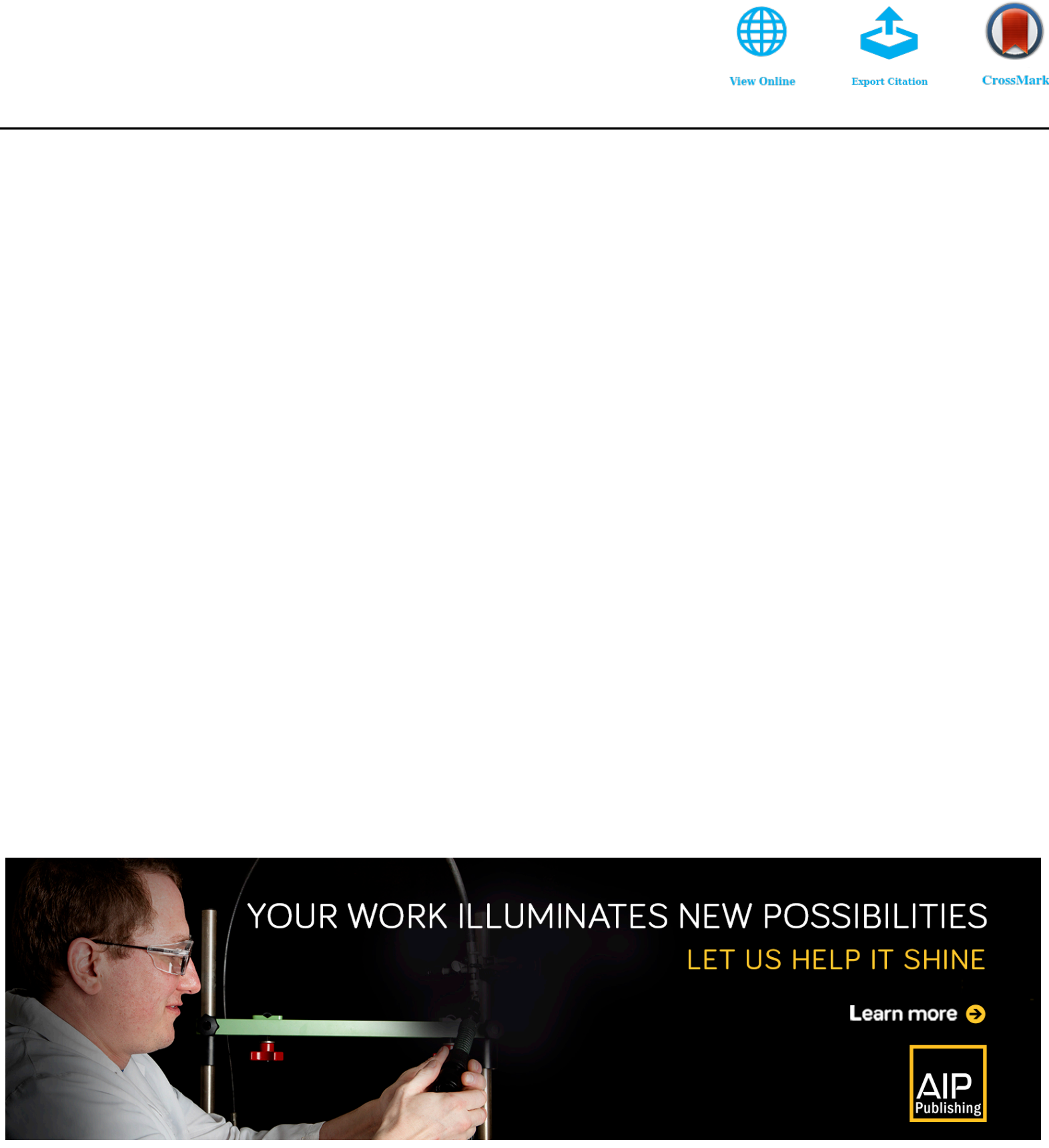


\title{
Development of a stagnation streamline model for thermochemical nonequilibrium flow
}

\author{
Cite as: Phys. Fluids 32, 046102 (2020); doi: 10.1063/5.0003247 \\ Submitted: 1 February 2020 - Accepted: 13 March 2020 • \\ Published Online: 1 April 2020
}

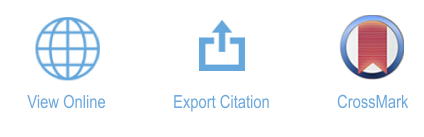

Qizhen Hong (洪启臻), ${ }^{1,2}$ (D) Xiaoyong Wang (王小永), (D) Yuan Hu (胡远), ${ }^{1}$ (D) and Quanhua Sun (孙泉华) ${ }^{1,2, a)}$ (D)

\begin{abstract}
AFFILIATIONS
'State Key Laboratory of High Temperature Gas Dynamics, Institute of Mechanics, Chinese Academy of Sciences, Beijing 100190, China

${ }^{2}$ School of Engineering Science, University of Chinese Academy of Sciences, Beijing 100049, China
\end{abstract}

a) Author to whom correspondence should be addressed: qsun@imech.ac.cn

\begin{abstract}
A stagnation streamline model incorporating quantum-state-resolved chemistry is proposed to study hypersonic nonequilibrium flows along the stagnation streamline. This model is developed by reducing the full Navier-Stokes equations to the stagnation streamline with proper approximations for equation closure. The thermochemical nonequilibrium is described by either the state-to-state approach for detailed analysis or conventional two-temperature models for comparison purpose. The model is validated against various data, and nearly identical results are obtained as compared with those from full field computational fluid dynamics data. In addition, the calculated distributions agree well with the measurement data of a shock tube experiment for the dissociation and vibrational relaxation of $\mathrm{O}_{2}$, including the distributions of species mole fractions and vibrational temperature of the first excited state of $\mathrm{O}_{2}$ molecules. Furthermore, the results with the stateresolved chemistry show that the flow within a shock layer exhibits a strong thermochemical nonequilibrium behavior, which is beyond the capability of commonly used two-temperature models to correctly evaluate the dissociation rate and the associated reaction energy. The present model is also employed to calculate the nonequilibrium re-entry flow along the stagnation streamline for a five-species air mixture as an example to demonstrate the model capability. It is found that both species and internal energy are in a nonequilibrium state, especially the vibrational distributions are strongly deviated from the Boltzmann distribution right behind the bow shock and near the wall surface. The results demonstrate that the proposed stagnation streamline model is very useful to understand thermochemical nonequilibrium phenomena in hypersonic flows.
\end{abstract}

Published under license by AIP Publishing. https://doi.org/10.1063/5.0003247

\section{INTRODUCTION}

Thermochemical nonequilibrium phenomena are very common in hypersonic flows, and a detailed analysis is still challenging. ${ }^{1-3}$ To design next generation hypersonic vehicles, it is important to develop reliable and fast computational tools to predict the flow characteristics. An accurate modeling requires one to handle detailed processes of chemical reactions and internal energy relaxations in the flow field. Parametric studies for engineering design demand extensive calculations over a wide range of flow conditions along the flight trajectory, and thus, numerical tools must be efficient.

For the past three decades, great efforts have been devoted to developing accurate models for thermochemical nonequilibrium hypersonic flows, and extraordinary progress has been achieved. ${ }^{3}$ The multi-temperature models have been the main tool to treat thermal nonequilibrium in hypersonic flows. ${ }^{4,5}$ In a multitemperature model, each internal energy mode follows a Boltzmann distribution with its equilibrium temperature, considering fast intramode transitions; thus, the model's applicability is limited to the situation where the intramode distribution is only slightly deviated from its own equilibrium. Unfortunately, this is often not the case for hypersonic flows. ${ }^{3}$ In order to extend the applicability of multi-temperature models, various corrections have been applied for reaction rate calculation. ${ }^{5-8}$ However, the model accuracy depends strongly on the choice of the empirical parameters in the corrections. More importantly, corrections in the multi-temperature models are not universally 
applicable when a flow is in a moderate or strong nonequilibrium state.

In order to describe strong nonequilibrium processes in hypersonic flows, state-to-state (STS) based models have been developed. $^{9-15}$ In a model based on STS kinetics, each internal energy level is treated as a pseudo-species, and thereby non-Boltzmann distribution can be represented. The transition processes between internal energy levels and chemical reactions are treated as elementary reaction processes. The key for STS simulations is to get the correct values of rate coefficients for the state transitions and chemical reactions. These coefficients are usually obtained from quasi-classical trajectory (QCT) calculations, which depend on the availability of the $a b$ initio potential energy surface (PES) for the molecular pairs of interest. While a database of $a b$ initio based rate coefficients for many elementary reactions of nitrogen and oxygen has been established, ${ }^{16-22}$ many reaction data are still missing. For reactions without $a b$ initio data, one usually resorts to approximate models, e.g., the Landau-Teller model, ${ }^{23,24}$ Schwartz-Slawsky-Herzfeld (SSH) theory, ${ }^{25}$ Forced Harmonic Oscillator (FHO) model, and its variations. $^{26-28}$ A typical simulation with a STS model needs to solve a system of hundreds of equations simultaneously because the number of state-to-state transitions is very large. Due to considerable computational cost, STS based applications are so far limited to simple one-dimensional (1D) geometries, including post-shock flows, ${ }^{29,30}$ nozzle flows, ${ }^{31}$ and boundary layer flows. ${ }^{14,32,33}$

In contrast to the considerable amount of work on thermochemical nonequilibrium models, development of high fidelity reduced models attracts much less attention. In fact, the nonequilibrium flow along the stagnation streamline usually contains thermochemical characteristics for the flow over blunt bodies because key physics such as the shock wave, shock layer, and boundary layer are all involved. One attempt was made by Klomfass and Müller, who proposed a dimension-reduced model for the fast assessment of hypersonic flow properties along the stagnation streamline. ${ }^{34} \mathrm{~A}$ coarse-grained rovibrational collisional model was later incorporated into the reduced model to study the nonequilibrium population of $\mathrm{N}_{2}$ along the stagnation streamline. ${ }^{35}$ The main drawback of this model is that a newtonian-like pressure is assumed along the streamline, so it holds only for high Mach flows or within small shock stand-off distances. Recently, Chen and Sun developed a quasi-one-dimensional model to predict flows along the stagnation streamline. $^{36}$ That model solves a set of partial differential equations derived from full Navier-Stokes (NS) equations, and its solution is nearly identical to full-field computational fluid dynamics (CFD) calculation. However, equations derived by Chen and Sun $^{36}$ are based on perfect gas, which needs to be extended to nonequilibrium gas mixture.

The present study extends the quasi-one-dimensional model developed by Chen and $\operatorname{Sun}^{36}$ to hypersonic thermochemical nonequilibrium flows of gas mixture by integrating both multitemperature models and quantum based STS kinetics. The new model coupling with the STS approach is then employed to investigate hypersonic thermochemical nonequilibrium flows of $\mathrm{O}_{2}-\mathrm{O}$ and $\mathrm{N}_{2} / \mathrm{N} / \mathrm{O}_{2} / \mathrm{O} / \mathrm{NO}$ gas mixtures along the stagnation streamline. The rest of the paper is organized as follows. First, nonequilibrium three-dimensional Navier-Stokes equations are described, and then the reduced model along the stagnation streamline for nonequilibrium flows is proposed in Sec. II. The model is validated in Sec. III, where full field CFD results and experimental data are compared. In Sec. IV, a couple of applications are presented, which shows detailed vibrational distributions of molecules along the streamline and reveals limitations of two-temperature models. Section $V$ gives some concluding remarks.

\section{STAGNATION STREAMLINE MODEL FOR NONEQUILIBRIUM FLOW}

When the Knudsen number is sufficiently small, the NavierStokes equations are adequate to describe thermochemical nonequilibrium flows if real gas effects are properly taken into account. The thermochemical processes can be modeled by either the state-tostate (STS) approach or multi-temperature models. In this section, the governing equations of three-dimensional nonequilibrium flows will be first described, and a reduced model will then be presented for the flow along the stagnation streamline.

\section{A. Nonequilibrium three-dimensional Navier-Stokes equations}

In the NS framework, the nonequilibrium thermochemical processes are best described using the STS approach where each internal energy level is treated as a pseudo-species. In the present work, the STS approach is only applied to the vibrational energy mode because vibrational nonequilibrium plays the most important role in many hypersonic flows. There is no difficulty in principle to apply the STS approach to other energy modes. The governing equations for three-dimensional (3D) nonequilibrium system can be written as ${ }^{9}$

$$
\frac{\partial Q}{\partial t}+\frac{\partial\left(F-F_{v}\right)}{\partial x}+\frac{\partial\left(G-G_{v}\right)}{\partial y}+\frac{\partial\left(W-W_{v}\right)}{\partial z}=S,
$$

where

$$
\begin{gathered}
Q=\left\{\begin{array}{c}
\rho_{c i} \\
\rho u \\
\rho v \\
\rho w \\
E
\end{array}\right\}, \quad S=\left\{\begin{array}{c}
\dot{\omega}_{c i} \\
0 \\
0 \\
0 \\
0
\end{array}\right\}, \quad \text { and } F=\left\{\begin{array}{l}
\rho_{c i} u \\
\rho u^{2}+p \\
\rho u v \\
\rho u w \\
(E+p) u
\end{array}\right\}, \\
F_{v}=\left\{\begin{array}{l}
-J_{x, c i} \\
\tau_{x x} \\
\tau_{x y} \\
\tau_{x z} \\
\tau_{x x} u+\tau_{x y} v+\tau_{x z} w-q_{t r, x}-\sum_{c i} J_{x, c i} h_{c i}
\end{array}\right\} .
\end{gathered}
$$

In these expressions, $\rho_{c i}$ is the mass density of molecular species $c$ at the $i$ th vibrational level (for atomic species, $\rho_{c i}$ is replaced with $\rho_{c}$ ), $u, v$, and $w$ are three bulk velocity components, and $E$ and $h_{c i}$ are the total energy and species enthalpy per unit mass, respectively. $\dot{\omega}_{c i}$ is the mass production rate of species $c$ at the $i$ th vibrational level due to inelastic collisions and chemical reactions. Moreover, $p$ denotes the pressure calculated by Dalton's law, i.e., $p=\bar{R} T \sum_{j=1}^{n s} \frac{\rho_{j}}{M_{j}}$, where $\bar{R}$ is the universal gas constant and $M_{j}$ is the molecular weight of species $j . \tau_{i j}$ is the $i j$ component of viscous stress and $q_{t r, x}$ is the 
translational-rotational heat flux in the $x$ th direction, and their expressions can be found in Ref. 9 where the modified ChapmanEnskog approximation was used. $J_{x, c i}$ is the species diffusion flux in the $\mathrm{x}$-direction. The flux vectors in the other two directions are similar, which is omitted here.

The essence of the STS approach is to calculate the value of mass production rate $\dot{\omega}_{c i}$ in Eq. (2) for every internal energy level based on the master equation, which can be expressed as

$$
\dot{\omega}_{c i}=\dot{\omega}_{c i}^{\mathrm{vib}}+\dot{\omega}_{c i}^{2 \rightleftharpoons 2}+\dot{\omega}_{c i}^{2 \rightleftharpoons 3}
$$

where $\dot{\omega}_{c i}^{\mathrm{vib}}, \dot{\omega}_{c i}^{2 \rightleftharpoons 2}$, and $\dot{\omega}_{c i}^{2 \rightleftharpoons 3}$ are the mass productions due to the vibration-translation $(\mathrm{V}-\mathrm{T})$ and vibration-vibration $(\mathrm{V}-\mathrm{V})$ transitions, the exchange reactions (EXs), and the dissociationrecombination $(\mathrm{D}-\mathrm{R})$ reactions, respectively. Details are given as follows:

$$
\begin{gathered}
\dot{\omega}_{c i}^{\mathrm{vib}}=\sum_{d k i^{\prime} k^{\prime}}\left(n_{c i^{\prime}} n_{d k^{\prime}} k_{c, i^{\prime} i}^{d, k^{\prime} k}-n_{c i} n_{d k} k_{c, i i^{\prime}}^{d, k k^{\prime}}\right), \\
\dot{\omega}_{c i}^{2 \rightleftharpoons 2}=\sum_{d c^{\prime} d^{\prime}} \sum_{k i^{\prime} k^{\prime}}\left(n_{c^{\prime} i^{\prime}} n_{d^{\prime} k^{\prime}} k_{c^{\prime} i^{\prime}, c i}^{d^{\prime} k^{\prime}, d k}-n_{c i} n_{d k} k_{c i, c^{\prime} i^{\prime}}^{d k, d^{\prime} k^{\prime}}\right), \\
\dot{\omega}_{c i}^{2 \rightleftharpoons 3}=\sum_{d k} n_{d k}\left(n_{c^{\prime}} n_{f^{\prime}} k_{\mathrm{rec}, c i}^{d k}-n_{c i} k_{\mathrm{dis}, c i}^{d k}\right) .
\end{gathered}
$$

In Eqs. (5)-(7), $k_{c, i^{\prime} i}^{d, k^{\prime} k}$ is the rate coefficient for the transitions between vibrational energy levels (including $\mathrm{V}-\mathrm{T}$ and $\mathrm{V}-\mathrm{V}$ ); $k_{c^{\prime} i^{\prime}, c i}^{d^{\prime}, d k}, k_{\mathrm{dis}, c i}^{d k}$, and $k_{\mathrm{rec}, c i}^{d k}$ are the rate coefficients for the exchange, dissociation, and recombination reactions, respectively. The state-specific rate coefficients of these transitions and chemical reactions are usually taken from the results of quasi-classical ${ }^{17,18}$ and semi-classical ${ }^{37,38}$ numerical trajectory calculations or analytic methods (such as the FHO model $^{28}$ ).

The number of vibrational levels for a molecule can be several tens or even hundreds, and then the number of possible state-tostate transitions can be as high as tens of thousands. This means that one may have to solve hundreds of master equations involving tens of thousands reactions if the STS approach is employed. Therefore, a full 3D STS NS simulation is prohibitively computationally expensive even with the most powerful supercomputer. To alleviate the extremely high cost of full STS simulations, one has to resort to simplified models.

Multi-temperature models [especially two-temperature (2T) model $\left.{ }^{5-7}\right]$ are more effective computationally than the STS approach. In a multi-temperature model, it is assumed that the internal energy levels follow a quasi-stationary distribution. Specifically, the two-temperature model of Park ${ }^{5}$ assumes that the translational energy and rotational energy of a gas mixture are at an equilibrium distribution of a temperature $T_{t r}$, whereas the vibrational energy and electronic energy are described with another temperature $T_{v e}$. In a simulation using the two-temperature model, one does not need to resolve every single vibrational energy state but need to solve only one equation for the vibrational-electronic energy instead. Then, the terms in the governing equations, Eq. (1), become ${ }^{39}$

$$
\begin{gathered}
Q=\left\{\begin{array}{c}
\rho_{c} \\
\rho u \\
\rho v \\
\rho w \\
E \\
E_{v e}
\end{array}\right\}, \quad S=\left\{\begin{array}{c}
\dot{\omega}_{c} \\
0 \\
0 \\
0 \\
0 \\
\Omega_{v e}
\end{array}\right\}, \quad \text { and } F=\left\{\begin{array}{l}
\rho_{c} u \\
\rho u^{2}+p \\
\rho u v \\
\rho u w \\
(E+p) u \\
E_{v e} u
\end{array}\right\}, \\
F_{v}=\left\{\begin{array}{l}
-J_{x, c} \\
\tau_{x x} \\
\tau_{x y} \\
\tau_{x z} \\
\tau_{x x} u+\tau_{x y} v+\tau_{x z} w-q_{t r, x}-q_{v e, x}-\sum_{c} J_{x, c} h_{c} \\
-q_{v e, x}-\sum_{c} J_{x, c} e_{v e, c}
\end{array}\right\},
\end{gathered}
$$

where $E_{v e}$ is the vibrational-electronic energy per unit volume of mixture. $\Omega_{v e}$ is the vibrational-electronic energy source term. $q_{t r, x}$ and $q_{v e, x}$ are the translational-rotational and vibrational-electronic heat fluxes in the $x$ th direction, respectively. The flux vectors in the other two directions are similar. It should be mentioned that the energy source terms $\Omega_{v e}{ }^{5-7}$ includes the relaxation energy source terms $\left(\Omega_{v-t}, \Omega_{e-t}\right)$ and reactive source term $\left(\Omega_{v-d}, \Omega_{e-I}\right)$. The details of Park's two-temperature model are described in Appendix A.

Despite their success in many applications, two-temperature models suffer from several deficiencies. A two-temperature model usually includes case-dependent empirical tuning parameters. Moreover, the assumption of Boltzmann distribution is questionable for flows with strong vibrational excitations and dissociation. Therefore, the STS approach is required if one's top priority is the high accuracy of numerical results for strongly thermochemical nonequilibrium flows. As a 3D full-field STS simulation is too expensive computationally to be realized for practical engineering applications, a high-fidelity reduced model is, therefore, preferred for nonequilibrium flow along the stagnation streamline.

\section{B. Nonequilibrium stagnation streamline model}

As mentioned in Sec. II A, it is very expensive numerically to apply the STS approach to study the entire flow field around a hypersonic vehicle. Then, the strategy is to find an important part of the flow for the STS approach to investigate. For hypersonic flow over a blunt body, the variation of flow properties is probably most prominent along the stagnation streamline. It has been shown that the flow along the stagnation streamline of a blunt body can be studied using the quasi-one-dimensional Navier-Stokes equations. ${ }^{36}$ It is then beneficial to develop a stagnation streamline model with the STS approach; thus, the major thermochemical nonequilibrium processes can be well studied for hypersonic flow over blunt bodies.

In order to derive the governing equation along the stagnation streamline, the three-dimensional nonequilibrium Navier-Stokes equations are first rewritten in spherical coordinates $(r, \theta, \varphi$; see Fig. 1). Then, considering the feature of spherical-symmetric in the vicinity of the stagnation streamline, on the stagnation line $(\theta \rightarrow 0)$, we have

$$
u=\sin \theta \frac{\partial u}{\partial \theta},\left.\frac{\partial \phi}{\partial \theta}\right|_{\phi \neq u}=0
$$




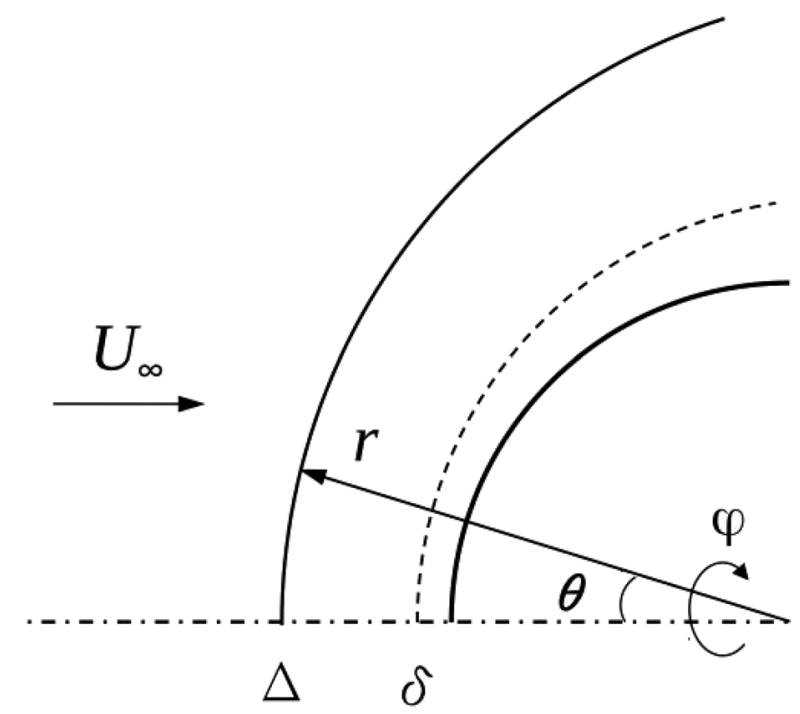

FIG. 1. Schematic diagram of hypersonic flow over a blunt body in the spherical coordinate.

where $u$ is the velocity along the $\theta$ direction. Moreover, it is reasonable to assume that

$$
\frac{1}{r^{2}} \frac{\partial^{2} v}{\partial \theta^{2}} \ll \frac{\partial^{2} v}{\partial r^{2}}, \frac{1}{r^{2}} \frac{\partial^{2} T_{t r, v e}}{\partial \theta^{2}} \ll \frac{\partial^{2} T_{t r, v e}}{\partial r^{2}},
$$

where $v$ is the velocity along the $r$ direction and $T_{t r, v e}$ are translational-rotational and vibrational-electronic temperatures, respectively. ${ }^{4}$ Under these assumptions and steady state condition, a set of ordinary differential equations can be derived from the full nonequilibrium Navier-Stokes equations [Eq. (1)]. Specifically, the equations of the stagnation streamline model based on the two-temperature model are written as follows:

Continuity equation:

$$
\frac{\partial v}{\partial r}+\frac{v}{\rho} \frac{\partial \rho}{\partial r}+\frac{2}{r} \frac{\partial u}{\partial \theta}+\frac{2}{r} v=0
$$

Mass conservation equation for each species:

$$
\rho v \frac{\partial y_{c}}{\partial r}-\frac{\partial}{\partial r}\left(\rho D_{c} \frac{\partial y_{c}}{\partial r}\right)-\frac{2 \rho D_{c}}{r} \frac{\partial y_{c}}{\partial r}=\dot{\omega}_{c}, \quad c=1, \ldots, n s,
$$

where $y_{c}=\rho_{c} / \rho$ is the mass fraction of species $c, n s$ is the total number of species, and $D_{c}$ is the diffusion coefficient of species $c$ in Fick's law. ${ }^{40}$

Momentum equation along the $r$ direction:

$$
\begin{aligned}
\rho v \frac{\partial v}{\partial r}+\frac{\partial p}{\partial r}= & 2 \frac{\partial \mu}{\partial r}\left(\frac{\partial v}{\partial r}+\frac{v}{3 \rho} \frac{\partial \rho}{\partial r}\right)+\mu\left(\frac{\partial^{2} v}{\partial r^{2}}+\frac{2}{r^{2}} \frac{\partial^{2} v}{\partial \theta^{2}}+\frac{4}{r} \frac{\partial v}{\partial r}+\frac{2 v}{r^{2}}\right. \\
& \left.-\frac{1}{3 \rho} \frac{\partial v}{\partial r} \frac{\partial \rho}{\partial r}+\frac{2 v}{r \rho} \frac{\partial \rho}{\partial r}-\frac{v}{3 \rho} \frac{\partial^{2} \rho}{\partial r^{2}}+\frac{v}{3 \rho^{2}} \frac{\partial \rho}{\partial r} \frac{\partial \rho}{\partial r}\right)
\end{aligned}
$$

where $\mu$ is the viscosity of the mixture.

Momentum equation along the $\theta$ direction:

$$
\begin{aligned}
\rho\left[v \frac{\partial}{\partial r}\left(r \frac{\partial u}{\partial \theta}\right)+\frac{\partial u}{\partial \theta} \frac{\partial u}{\partial \theta}\right]= & r^{2} \frac{\partial}{\partial r}\left(\frac{\partial u}{r \partial \theta}\right) \frac{\partial \mu}{\partial r}+\mu\left(r \frac{\partial^{2}}{\partial r^{2}} \frac{\partial u}{\partial \theta}\right. \\
& \left.+2 \frac{\partial}{\partial r} \frac{\partial u}{\partial \theta}-\frac{1}{r} \frac{\partial u}{\partial \theta}\right)-\frac{\partial^{2} p}{\partial \theta^{2}}
\end{aligned}
$$

The conservation equation of enthalpy is

$$
\begin{aligned}
\rho v \frac{\partial h}{\partial r}-v \frac{\partial p}{\partial r}= & \mu\left(3 \frac{\partial v}{\partial r} \frac{\partial v}{\partial r}+\frac{2 v}{\rho} \frac{\partial v}{\partial r} \frac{\partial \rho}{\partial r}+\frac{v^{2}}{3 \rho^{2}} \frac{\partial \rho}{\partial r} \frac{\partial \rho}{\partial r}\right) \\
& +\sum_{i=t r, v e}\left(k_{i} \frac{\partial^{2} T_{i}}{\partial r^{2}}+k_{i} \frac{2}{r} \frac{\partial T_{i}}{\partial r}+\frac{\partial k_{i}}{\partial r} \frac{\partial T_{i}}{\partial r}\right) \\
& +\left(\frac{\partial}{\partial r}+\frac{2}{r}\right)\left(\sum_{c=\text { species }} h_{c} \rho D_{c} \frac{\partial y_{c}}{\partial r}\right),
\end{aligned}
$$

where $k_{t r}$ is the thermal conductivity ascribed to translational and rotational energy modes and $k_{v e}$ is the thermal conductivity ascribed to vibrational and electronic energy modes.

The equation of vibrational-electronic energy is

$$
\begin{aligned}
\rho v \frac{\partial e_{v e}}{\partial r}= & \frac{\partial}{\partial r}\left(k_{v e} \frac{\partial T_{v e}}{\partial r}\right)+\frac{2 k_{v e}}{r} \frac{\partial T_{v e}}{\partial r} \\
& +\left(\frac{\partial}{\partial r}+\frac{2}{r}\right)\left(\sum_{c=s p e c i e s} h_{c, v e} \rho D_{c} \frac{\partial y_{c}}{\partial r}\right)+\Omega_{v e}
\end{aligned}
$$

where $h_{c, v e}$ is the vibrational-electronic enthalpy per unit mass of species $c$.

So far, the complete set of reduced order equations for the twotemperature model is described. When it comes to the STS approach, there is no need to solve a separate vibrational energy equation since each quantized state is treated as a separate chemical species. The source terms in the species continuity equations are more complicated and time-consuming than those in two-temperature models. Other treatments for the STS based model are, however, similar. Details of state-specific quasi-one-dimensional equations are provided in Appendix B.

As the flow near the stagnation line is axial symmetric, the circumferential velocity is zero on the stagnation line. The radial velocity might be derived using the continuity equation [i.e., Eq. (12)], where $\partial u / \partial \theta$ is to be estimated using the momentum equation in the circumferential direction [i.e., Eq. (15)]. However, it is difficult to evaluate those involved second order gradients along the circumferential direction in Eq. (15), simplifications should be made to close the equations. For instance, in order to estimate the second order circumferential derivative of pressure, many attempts have been done. ${ }^{34,35,41,42}$ Nevertheless, the involved second order gradients should not be simply ignored or replaced by approximation theories such as the variable separation method and Newtonian theory $^{34,35,41,43}$ as the results have obvious deviation from CFD data. Fortunately, the radial mass flux along the stagnation streamline is found to decrease linearly from the bow shock to the stagnation point. ${ }^{42}$ This observation can be expressed as

$$
\rho v=\rho_{s} v_{s} r / \Delta,
$$


where $\Delta$ is the shock stand-off distance and subscript $s$ denotes the values immediately behind the shock wave. The shock wave is assumed to be infinitely thin, and the shock stand-off distance has to be estimated to close the equations. There have been many studies on the shock stand-off distance, ${ }^{44,45}$ and the formula proposed by Wen and Hornung ${ }^{44}$ is found to work well for inviscid reacting flows. For viscous flows, the thickness of the boundary layer $(\delta)$ can be estimated using the expression suggested by Wang. ${ }^{46}$ Then, the shock stand-off distance for the viscous flow is given as

$$
\frac{\Delta}{2 R_{0}}=0.41 \frac{\rho_{\infty}}{\bar{\rho}}+c_{1}\left(1+\frac{T_{b}}{T_{0}}\right)^{\omega / 2} \frac{M a_{\infty}^{\omega}}{\sqrt{R e_{\infty}}},
$$

where $\bar{\rho}$ is the average density from the bow shock to the stagnation point, $R_{0}$ is the nose radius of the blunt body, $\omega$ is the temperature index of viscosity, $T_{b}$ is the surface temperature, $T_{0}$ is the total temperature of free stream, and $c_{1}$ equals to 0.12 . It should be mentioned that thermochemical effects have been included in Eq. (19) through the average density. With Eq. (19), there is no need to solve the momentum equation along the circumferential direction. Then, the governing equations along the stagnation streamline become ordinary differential equations, which can be discretized using the finite difference method and be easily solved.

To obtain the solution along the stagnation streamline, boundary conditions are to be set. On the surface of the blunt body (namely, the stagnation point), the non-slip condition for velocity, zero gradient of pressure, and isothermal assumption are applied. For surface reactions, non-catalytic or fully catalytic wall conditions can be set according to surface physics. On the bow shock, the Rankine-Hugonoit relation can be used to set the post-shock conditions. However, considering the flow gradients along the stagnation streamline, the shock slip conditions are applied to determine the boundary condition behind the shock wave. ${ }^{47}$ The dimensionless shock slip conditions are

$$
\begin{gathered}
v_{s}^{*}=1 / \rho_{s}^{*}, \\
p_{s}^{*}=p_{\infty}^{*}+\left(1-\frac{1}{\rho_{s}^{*}}\right)+\varepsilon^{2} \frac{4}{3}\left(\mu_{s}^{*} \frac{\partial v_{s}^{*}}{\partial r^{*}}\right) \\
h_{t r, \infty}^{*}-h_{t r, s}^{*}+\frac{1}{2}\left(1-v_{s}^{* 2}\right)=\varepsilon^{2} k_{t r, s}^{*} \frac{\partial T_{t r, s}^{*}}{\partial r^{*}}+\varepsilon^{2} \rho_{s}^{*}\left(\sum_{c=s p e c i e s} h_{t r, c}^{*} D_{c}^{*} \frac{\partial y_{c}}{\partial r^{*}}\right)_{s} \\
+\frac{4}{3} \varepsilon^{2} v_{s}^{*} \mu_{s}^{*} \frac{\partial v_{s}^{*}}{\partial r^{*}}, \\
h_{v e, \infty}^{*}-h_{v e, s}^{*}=\varepsilon^{2} \rho_{s}^{*}\left(\sum_{c=s p e c i e s} h_{c, v e}^{*} D_{c}^{*} \frac{\partial y_{c}}{\partial r^{*}}\right)_{s}+\varepsilon^{2} k_{\mathrm{ve}, s}^{*} \frac{\partial T_{\mathrm{ve}, s}^{*}}{\partial r^{*}}
\end{gathered}
$$

where ${ }^{*}$ represents dimensionless quantity, $\varepsilon^{2}=\frac{1}{\operatorname{Re}_{\infty}}$, and $R e_{\infty}$ is the free stream Reynolds number. The above expressions take into account the viscous effects and give more accurate post-shock flow conditions than the Rankine-Hugoniot relation does.

\section{VALIDATION}

The present stagnation streamline model can be solved numerically with very high spatial resolution within several minutes on a current personal computer. The solution using this model is expected to agree with the corresponding results obtained from a full-field CFD solution along the stagnation streamline. Such comparisons are made extensively where the CFD data are obtained either using an in-house CFD solver or from the literature. ${ }^{48}$ Other comparisons are also made with the results from the dimensionally reduced Navier-Stokes equations (DRNSEs) ${ }^{34}$ and with the measurement data ${ }^{49}$ of a shock tube experiment.

For full-field CFD solution using the in-house CFD code, the nonequilibrium Euler or Navier-Stokes equations are discretized on multi-block structured meshes using the finite volume method. The inviscid fluxes are evaluated using Roe's upwind scheme with second order MUSCL (monotonic upstream scheme for scalar conservation law) reconstruction and minmod limiter, and the viscous fluxes are calculated using the second order central difference scheme. The Data-Parallel Line Relaxation (DPLR) method is employed for time marching. For Navier-Stokes solutions, the viscosity of each species is calculated using kinetic theory with collision integral, whereas the thermal conductivity is calculated using the modified Eucken relation. Fick's law is assumed for mass diffusion, and the Lewis number is fixed at 1.4. The reaction rate constants are calculated according to the Arrhenius-type correlations of Park. ${ }^{4}$ Overall, same thermochemical models and transport models are set for both full CFD simulations and stagnation streamline modeling. For each comparison, mesh independent results are obtained by refining structured meshes for CFD simulation, whereas over 1000 grid points are used for the stagnation streamline model to obtain fine results.

In the following test cases, the air mixture of $\mathrm{O}_{2}$ and $\mathrm{N}_{2}$ flows over a sphere with a radius of $0.1 \mathrm{~m}$ at a Mach number of $10(\mathrm{Ma}$ $=10)$. The free stream conditions are set according to the standard atmospheric condition at different altitudes, and five species $\left(\mathrm{O}_{2}, \mathrm{~N}_{2}, \mathrm{NO}, \mathrm{O}\right.$, and $\left.\mathrm{N}\right)$ are considered to account for the thermochemical nonequilibrium processes. Figure 2 shows the comparison between the stagnation streamline model and CFD simulation for the inviscid flow at a height of $40 \mathrm{~km}(H=40 \mathrm{~km})$ and $53 \mathrm{~km}(H$ $=53 \mathrm{~km}$ ), respectively. For both cases, the translational-rotational temperature $T$ decreases after the shock due to the conversion from translational to vibrational energy and the dissociation reaction. The vibrational temperature $T_{v}$ increases from the shock and approaches to the translational-rotational temperature along the streamline. The mass fraction of $\mathrm{O}\left(y_{\mathrm{O}}\right)$ also increases along the streamline due to the dissociation of $\mathrm{O}_{2}$. It is found that the shock stand-off distance predicted by Eq. (19) agrees well with the value from the CFD results. Moreover, Fig. 2 clearly shows that the present model predicts nearly identical flow properties as the full field CFD simulations, which indicates the validity of the present model for inviscid thermo-chemical nonequilibrium flows.

Figure 3 shows the comparison of viscous flow properties along the stagnation streamline for above two typical cases. Herein, the wall surface is non-catalytic and remains at $300 \mathrm{~K}$. Different from the inviscid cases, there is a thin boundary layer in the viscous case, where the temperature drops quickly to the surface temperature and the density rises accordingly. It is found that there is a maximum of $\mathrm{O}$ mass fraction in viscous flow, which is due to the recombination reactions dominated in the boundary layer. As the influence of the boundary layer has been taken into account, the empirical formula Eq. (19) predicts the shock stand-off distance very well in the 

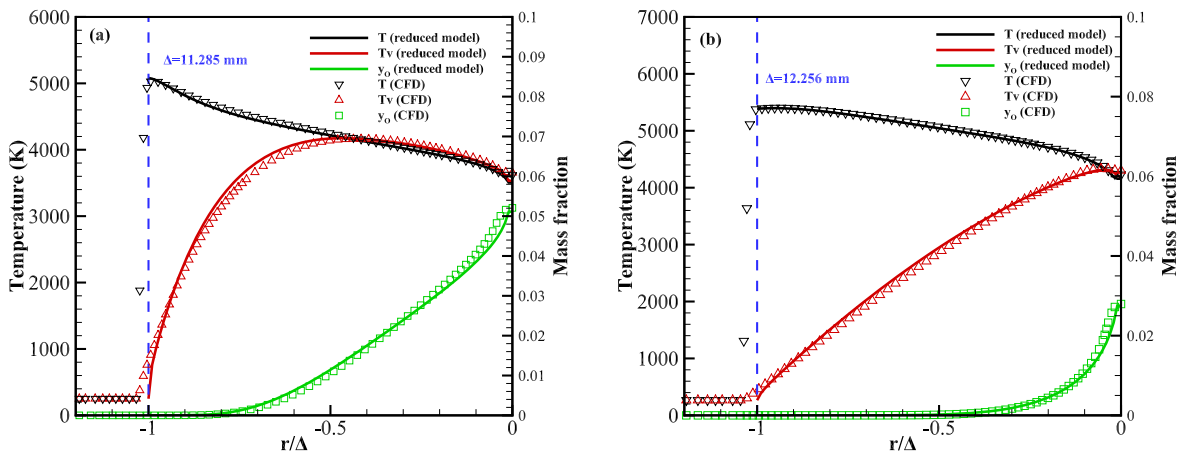

FIG. 2. Comparison of flow properties along the stagnation streamline for inviscid flow results at $M a=10$ between the present model and CFD simulation. (a) $H=40 \mathrm{~km}$ and (b) $H=53 \mathrm{~km}$.
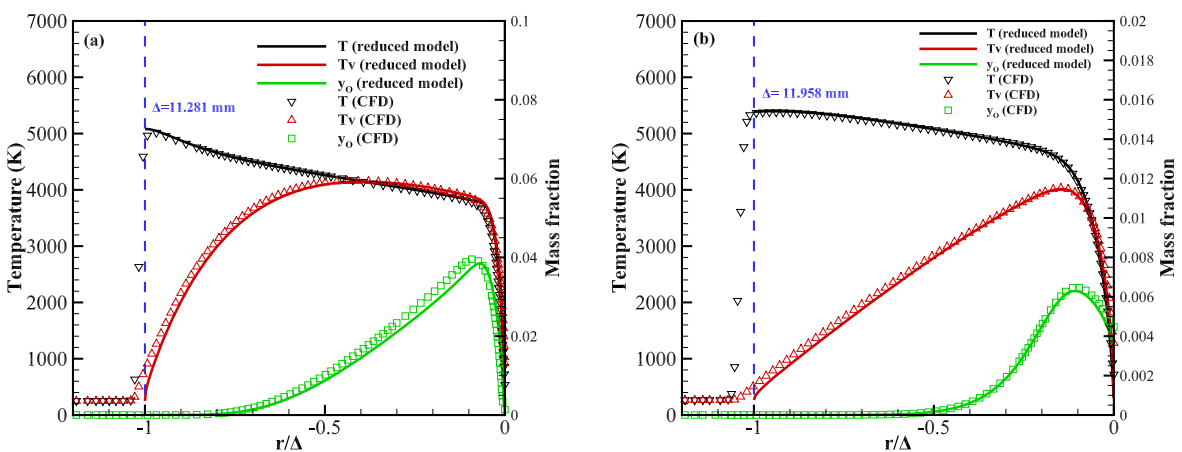

FIG. 3. Comparison of flow properties along the stagnation streamline for viscous flow results at $M a=10$ between the present model and CFD simulation. (a) $H=40 \mathrm{~km}$ and (b) $H=53 \mathrm{~km}$.

viscous flow. Again, Fig. 3 clearly shows that the present model predicts nearly identical flow properties as the full field CFD simulations. The present model is found to be valid for viscous thermochemical nonequilibrium flows.

In addition to comparisons with the results of the in-house CFD solver, detailed comparisons between the present model and the results of 3D full-field CFD in Ref. 48 are conducted. In Ref. 48, the following cases of hypersonic flow over a sphere with a radius of $1 \mathrm{~m}$ are investigated: (1) $h_{\infty}=7.2 \mathrm{MJ} / \mathrm{kg}, u_{\infty}=3750 \mathrm{~m} / \mathrm{s}$, $p_{\infty}=8.188 \mathrm{~Pa}, T_{\infty}=220 \mathrm{~K}, \quad y_{\mathrm{N}_{2}, \infty}=0.7381, y_{\mathrm{O}_{2}, \infty}=0.2619$ and (2) $h_{\infty}=25 \mathrm{MJ} / \mathrm{kg}, u_{\infty}=7070 \mathrm{~m} / \mathrm{s}, p_{\infty}=10.11 \mathrm{~Pa}, T_{\infty}$ $=220 \mathrm{~K}, \quad y_{\mathrm{N}_{2}, \infty}=0.7381, y_{\mathrm{O}_{2}, \infty}=0.2619$. In order to make the comparisons, the same thermochemical models and transport models in Ref. 48 are intentionally used for the calculation of the stagnation streamline model. As shown in Fig. 4, where the translational-rotational temperature $T$, vibrational temperature $T_{v}$, and the mass fraction of oxygen atom $y_{\mathrm{O}}$ are plotted along the stagnation streamline, the agreement between the present model results and the full field CFD simulations in Ref. 48 is satisfactory. Small discrepancies near the shock front are mainly caused by the shock capture method used in the full-field CFD simulation and insufficient grid resolution for shock wave in Ref. 48.

The validations discussed above demonstrate that one can confidently use the present stagnation streamline model to accurately reproduce $3 \mathrm{D}$ full field CFD results along the stagnation streamline. Moreover, the outperformance of the present stagnation streamline model is assessed by comparing with the results of Klomfass's dimensionally reduced Navier-Stokes equations (DRNSEs), 34,35,43
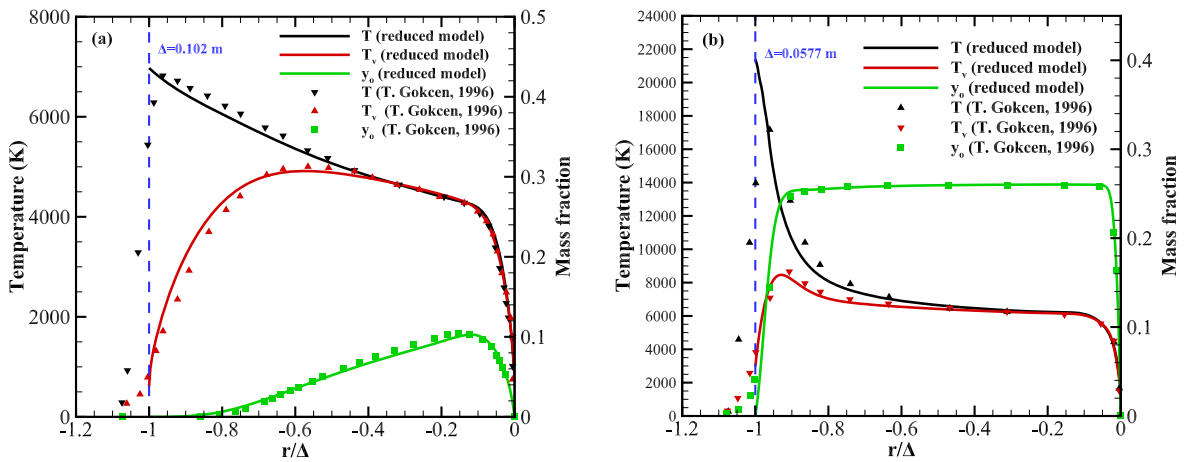

FIG. 4. Comparison of flow properties along the stagnation streamline for viscous flow between the present model and Gokcen's results. ${ }^{48}$ (a) The case of enthalpy $7.2 \mathrm{MJ} / \mathrm{kg}$. (b) The case of enthalpy $25 \mathrm{MJ} / \mathrm{kg}$. 

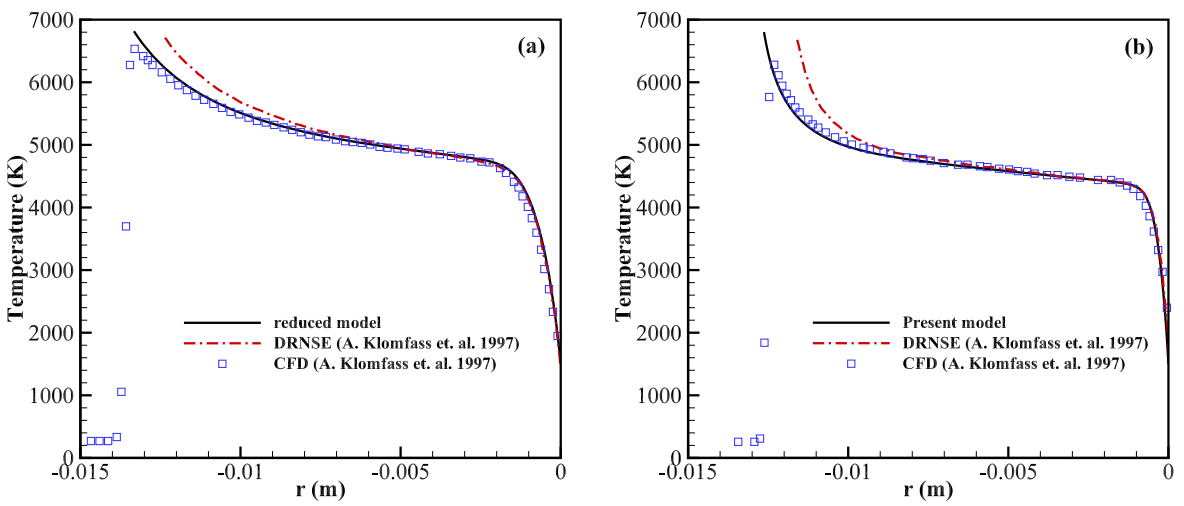

FIG. 5. Comparison of the temperature profile along the stagnation streamline between the present model and the results from Ref. 34: (a) Case 2.3 in Ref. 34 and (b) case 2.4 in Ref. 34. which is also a reduced-order model along the stagnation streamline. Figure 5 shows the results (of test cases 2.3 and 2.4 in Ref. 34) for the temperature obtained by different methods including the present model, Klomfass's model, and CFD. It is observed that the present stagnation streamline model gives results that are very close to the CFD results in Ref. 34 for both cases, whereas the DRNSE ${ }^{34}$ fails to predict both the temperature profile and the shock stand-off distance. The deviation of DRNSE results may be attributed to, as mentioned in Ref. 34, the assumption of a newtonian-like pressure distribution, which holds for high Mach numbers or small shock stand-off distances only. The outperformance of the present model than that of DRNSE suggests that the assumptions adopted in the present model are more reasonable and accurate.

The above results demonstrate the capability of the present model in simulating nonequilibrium flows using the two-temperature model. Furthermore, the stagnation streamline model coupled with the state-to-state approach is to be examined. The test case is adopted from ground experiments on the dissociation and vibrational relaxation of shock heated oxygen. ${ }^{49}$ Therein, vibrational temperature and concentration profiles of $\mathrm{O}$ behind shock front were measured via absorption spectroscopy studies in the Schumann Runge band with the post-shock translational temperature ranging from $4000 \mathrm{~K}$ to $10800 \mathrm{~K} .^{49}$ In our simulation, the flow past a blunt body is simulated using the stagnation streamline model and the wall is placed at the far-enough downstream. This is usually named as a blunt-body analogue of the $1 \mathrm{D}$ shock. ${ }^{50,51}$ In the simulation, the radius of the blunt body with a temperature of $300 \mathrm{~K}$ is set to be
$0.1 \mathrm{~m}$, which is long enough to have a large shock stand-off distance. A total of 47 vibrational levels proposed in Ref. 52 are considered for molecular oxygen in the ground electronic state. As for chemical kinetics, the $\mathrm{O}_{2}-\mathrm{O}_{2}$ dissociation and $\mathrm{V}-\mathrm{T}$ reaction rates are calculated using the FHO model as done in Ref. 30, the $\mathrm{O}_{2}-\mathrm{O}$ dissociation and $\mathrm{V}-\mathrm{T}$ reaction rates are calculated using the fitted QCT data formula, ${ }^{17}$ and finally, the $\mathrm{V}-\mathrm{V}-\mathrm{T}$ reaction rates are obtained using the simplified analytical FHO model.

The profiles of temperatures and species mole fractions of $\mathrm{O}_{2}(0$ $\rightarrow 46)$ and $\mathrm{O}$ are shown in Fig. 6 for the case of highest total enthalpy in the experiment, ${ }^{49}$ i.e., $V_{\infty}=4440 \mathrm{~m} / \mathrm{s}, \mathrm{P}_{\infty}=106.658 \mathrm{~Pa}$, and $\mathrm{T}_{\infty}$ $=295 \mathrm{~K}$. The evolution process of each vibrational state is depicted in detail in Fig. 6. On the upper panel, the vibrational temperature distributions of 1st, 10th, 20th, 30th, and 40th excited states are given along the stagnation streamline. The vibrational temperature of the particular excited state is defined as the number density ratio of this excited state to the ground state, i.e.,

$$
T_{v, i}=\frac{e_{v i b}(i)-e_{v i b}(0)}{k_{B} \ln \left(\left[\mathrm{O}_{2}(i)\right] /\left[\mathrm{O}_{2}(0)\right]\right)} .
$$

In Fig. 6(a), the average vibrational temperature is also plotted, representing the average energy under equilibrium, and is defined as

$$
\frac{\sum_{i} n_{i} e_{i, v i b}}{\sum_{i} n_{i}}=\frac{\sum_{i} e_{i, v i b} \exp \left(-e_{i, v i b} / k T_{v}\right)}{\sum_{i} \exp \left(-e_{i, v i b} / k T_{v}\right)} .
$$

It is seen from Fig. 6 that both mole fraction of $\mathrm{O}$ and vibrational temperature of the first excited state match well with the
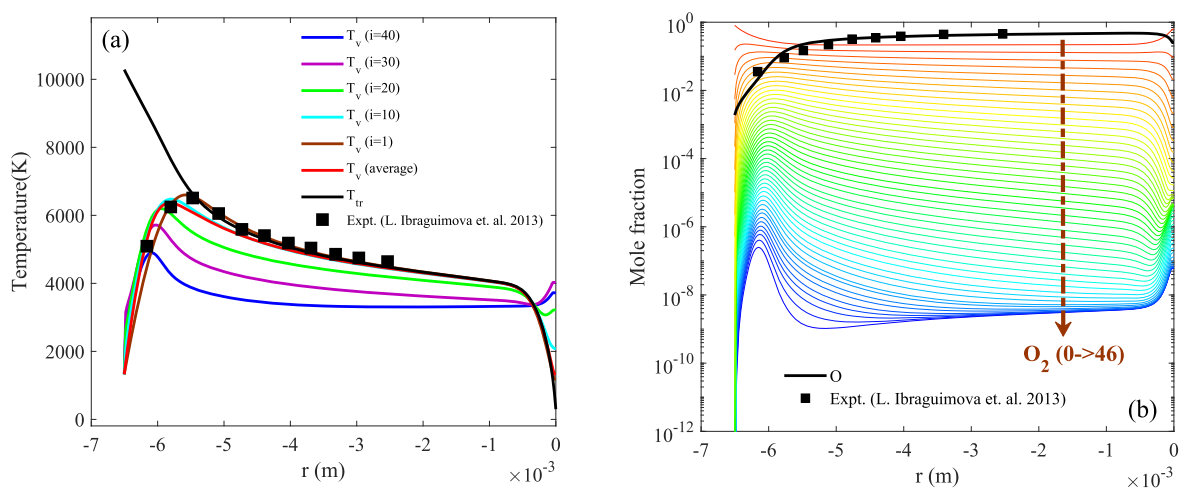

FIG. 6. Profiles of temperatures and species mole fractions of various vibrational energy levels along the stagnation streamline. (a) Vibrational temperatures of various excited vibrational levels, and translational and energy-presentative vibrational temperatures are also shown. (b) Mole fraction of $\mathrm{O}_{2}(0 \rightarrow 46)$ and $\mathrm{O}$. 
experimental measurements. It further validates the present stagnation streamline model. Further detailed analysis of thermochemical nonequilibrium in $\mathrm{O}_{2} / \mathrm{O}$ binary mixture will be presented in Sec. IV.

\section{APPLICATIONS}

Regarding hypersonic nonequilibrium flows, thermochemical behaviors are always associated with compressibility, viscous effect, and surface temperature. Individual simulations are required to understand each specific flow case, and a large number of simulations are desired to analyze the nature of thermochemical flows. Therefore, the stagnation streamline model is preferred as it is efficient and can be incorporated with advanced thermochemical models. In this section, two examples of hypersonic flows over a sphere are presented to demonstrate the capability of the stagnation streamline model for thermochemical analysis. The gas mixture in the first case is $\mathrm{O}_{2} / \mathrm{O}$, and the profiles of temperature and mole fraction of $\mathrm{O}$ have been discussed in Sec. III. The number density distribution of vibrational energy levels is presented in this section, which is used to check the validity of the two-temperature model. ${ }^{5}$ The gas mixture in the second case is five-species air mixture $\left(\mathrm{N}_{2} / \mathrm{N} / \mathrm{O}_{2} / \mathrm{O} / \mathrm{NO}\right)$. The flow condition is set at the altitude of $40 \mathrm{~km}$ with the Mach number of 10. In this case, detailed thermal chemistry is solved for hypersonic flow by resolving 157 vibrational states through 15201 state-to-state transitions along the stagnation streamline.

\section{A. Detailed analysis of thermochemical nonequilibrium in $\mathrm{O}_{2} / \mathrm{O}$ mixture}

Following the case of the $\mathrm{O}_{2} / \mathrm{O}$ binary mixture shown in Sec. III, the behavior of vibrational levels is analyzed here. As shown in Fig. 6, the level-specific vibrational temperatures increase rapidly after the bow shock due to the energy transferred from the translational energy ( $\mathrm{V}-\mathrm{T}$ energy transfer), and the excited vibrational levels make the number density of high levels increase. The mole fraction of $\mathrm{O}$ is also increased as the molecules keep dissociating. Furthermore, molecular dissociation is vibrationally favored; thus, the number density with a higher vibrational level decreases more rapidly. Therefore, a competition exists between the V-T energy transfer and molecular dissociation on the level-specific vibrational temperature, which results in a peak in the vibrational temperature at a certain distance downstream the bow shock. It is also observed from the temperature profile that molecules with lower vibrational levels are easier to reach the equilibrium state with the translational temperature although it takes longer time to reach their own peak. On the other side of the shock layer that is close to the wall surface, the low translational temperature leads to strong molecular recombination, which increases significantly the number density of molecules with high vibrational level. Thus, the level-specific vibrational temperatures of high energy levels rise again near the wall. The average vibrational temperature, however, decreases because the molecules with low vibrational energy are overwhelming due to the $\mathrm{V}-\mathrm{T}$ energy transfer from vibrational energy to low translational energy. This overall behavior of the vibrational nonequilibrium is similar to the normal shock case in Ref. 53 at the post-shock end and to the boundary layer case in Ref. 53 at the stagnation end.

In order to check the nonequilibrium deviation of the vibrational energy distribution along the stagnation streamline, several locations behind the shock front and close to the wall surface are selected, and the number density distribution of the vibrational levels is plotted in Fig. 7. Behind the shock front [see Fig. 7(a), $r=-6.3 \mathrm{~mm}$ ], the number density of high vibrational levels is significantly larger than the corresponding Boltzmann distribution in the logarithmic chart, which displays a state of "over-population." That is, a large number of molecules from low vibrational levels are transited to high vibrational levels via the V-T energy transfer. Meanwhile, molecular dissociation reduces the number density of molecules with very high vibrational energy. As the dissociation has not last long up to $r=-6.3 \mathrm{~mm}$, it is still dominated by the $\mathrm{V}-\mathrm{T}$ energy transfer. When the contribution from the vibrational temperature increase is overtaken by the dissociation rate, the number density of high vibrational levels drops below the corresponding Boltzmann distribution, which displays a state of "underpopulation" [as shown in the red and blue lines in Fig. 7(a), i.e., $r=-6.0 \mathrm{~mm}$ and $r=-5.0 \mathrm{~mm}$, respectively].

Close to the wall surface [see Fig. 7(b)], the recombination reaction occurs significantly because of the cold surface. At $r=-3.0 \mathrm{~mm}$, the vibrational level distribution is close to the Boltzmann distribution except that the number density of high level is a little higher. At $r=-2.0 \mathrm{~mm}$, the high level number density is much larger than the corresponding Boltzmann distribution, which indicates that the recombination reaction is dominated because molecules born from the recombination reaction is typically at very high vibrational level.
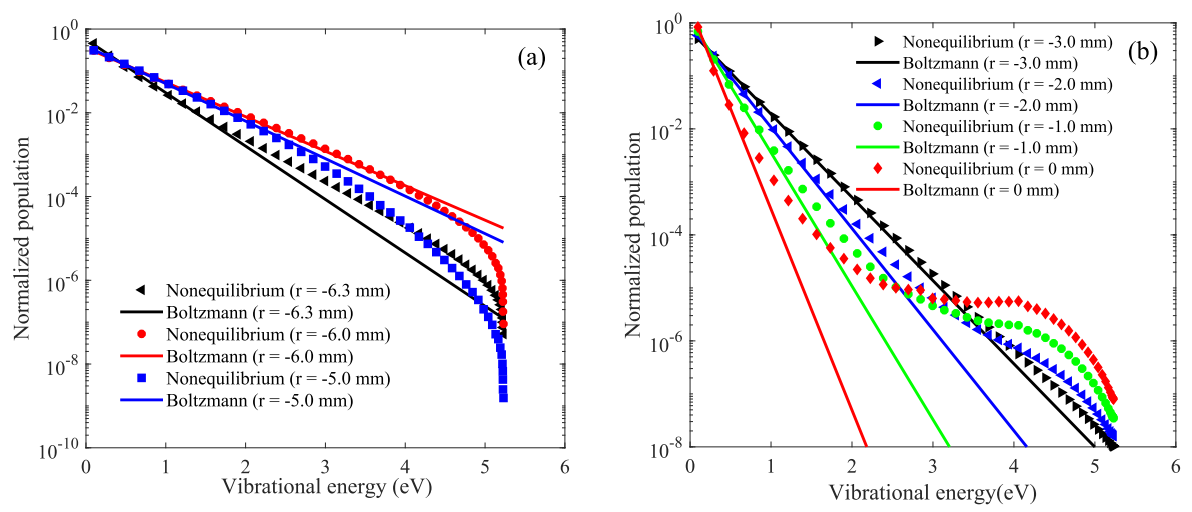

FIG. 7. Number density distributions of vibrational energy levels along the stagnation streamline. (a) Behind the shock front. (b) Close to the wall surface. 
The molecules having high vibrational energy will later be de-excited to lower vibrational level via molecular collisions. The de-excitation rate, however, is lower than the recombination rate; thus, the number density of molecules having very high vibrational level keeps increasing as the flow approaches the stagnation point. Moreover, there exists a plateau in the near wall vibrational distribution functions as a result of competition between de-excitation and recombination, which agrees with the results from the theoretical analysis in Ref. 33.

Based on the vibrational level resolved results of the state-tostate approach, the nonequilibrium dissociation rates and vibrational energy losses in dissociation are plotted along the stagnation streamline in Fig. 8. In Fig. 8(a), the macroscopic dissociation rate of $\mathrm{O}_{2}-\mathrm{O}_{2}$ and $\mathrm{O}_{2}-\mathrm{O}$ is obtained as the sum of dissociation rate of each vibrational state for the state-to-state results [Eq. (B9)], and they are calculated using Eq. (A4) with $q=0.5$ for the Park model. As Fig. 8(a) shows, the results of Eq. (A4) are different from those of Eq. (B9) not only in order of magnitude but also in the trend along the stagnation streamline. The non-monotonic behavior of the results of Eq. (B9) within the boundary layer comes from the fact that the very high energy states born from recombination reactions make great contributions to the nonequilibrium dissociation rate. The control temperature $\left(T_{a}=T_{t r}^{q} T_{v e}^{1-q}\right)^{5}$ for dissociation defined in the Park model accounts for the vibration-dissociation coupling only in two temperatures instead of nonequilibrium details, which fails to predict the dissociation rate phenomelogically. The vibrational energy losses in dissociation are presented in Fig. 8(b). For the Park model, it is too low to estimate the source term when assuming $e_{e f f}$ as the average vibrational energy $e_{v i b}$ because the dissociation reaction tends to occur at high vibrational states. It seems that the Park results agree well with the state-to-state results [Eq. (B10)] when the modeled vibrational energy in dissociation is assumed as 0.3 times of the molecular dissociation energy, which may explain the success of the Park model in some cases.

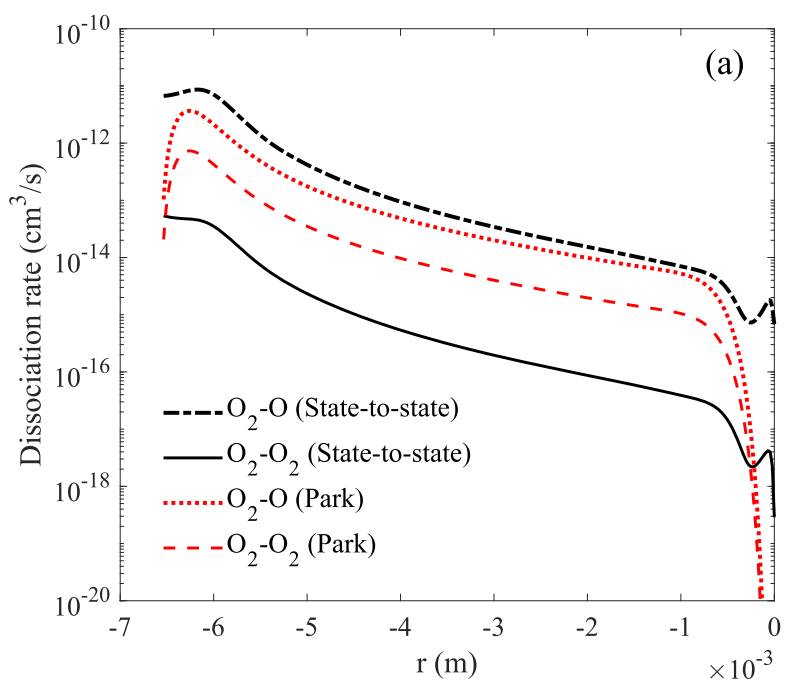

\section{B. State-to-state analysis of five-component air-mixture flow}

The previous case is about the flow of an $\mathrm{O}_{2} / \mathrm{O}$ mixture. For the realistic re-entry problem, the gas is air that is usually modeled as a five-component $\mathrm{N}_{2} / \mathrm{O}_{2} / \mathrm{N} / \mathrm{O} / \mathrm{NO}$ mixture. A state-to-state calculation of five-component air mixture for full field simulation would require large computational efforts that are not favorable for CFD solvers at the current stage. It is advantageous to employ the present stagnation streamline model to perform the state-specific simulation of air mixture and study related thermochemical nonequilibrium processes. In the present calculation, vibrational levels of diatomic molecules are taken from Ref. 54, i.e., $\mathrm{O}_{2}(0 \rightarrow 45), \mathrm{N}_{2}(0 \rightarrow 60)$, and $\mathrm{NO}(0 \rightarrow 47)$. The numbers in the parentheses are the vibrational states to be considered. Electronically excited states and free electrons are neglected because the focus of this paper is on the nonequilibrium of vibrational levels. The test case is similar to the previous case except the gas mixture, and the free stream condition is $M a=10$ and $H=40 \mathrm{~km}$.

For state-to-state simulation, three different categories of chemical processes [Eq. (4)] are included in the state-specific source term: the vibration-translation $(\mathrm{V}-\mathrm{T})$ energy transfer, dissociationrecombination (D-R), and Zeldovich exchange reaction (EX). The state-specific rates for above processes are mainly taken from the STELLAR database. ${ }^{55}$ Specifically, the results from QCT calculations are employed for $\mathrm{V}-\mathrm{T}$ and $\mathrm{D}-\mathrm{R}$ rates of $\mathrm{N}_{2}(\mathrm{v})+\mathrm{N}^{56}$ and $\mathrm{O}_{2}(\mathrm{v})+\mathrm{O} ;{ }^{57}$ the results from FHO model ${ }^{54,58}$ are employed for $\mathrm{V}-\mathrm{T}$ and $\mathrm{D}-\mathrm{R}$ rates of $\mathrm{N}_{2}(\mathrm{v})+\mathrm{N}_{2}, \mathrm{~N}_{2}(\mathrm{v})+\mathrm{O}_{2}, \mathrm{O}_{2}(\mathrm{v})+\mathrm{O}_{2}, \mathrm{O}_{2}(\mathrm{v})+\mathrm{N}_{2}$, and $\mathrm{NO}(\mathrm{v})$ $+\mathrm{N}_{2}$; the results from QCT calculations ${ }^{59,60}$ are employed for rates of two Zeldovich exchange reactions: $\mathrm{N}_{2}(\mathrm{v})+\mathrm{O} \rightleftarrows \mathrm{NO}(\mathrm{w})+\mathrm{N}$ and $\mathrm{O}_{2}(\mathrm{v})+\mathrm{N} \rightleftarrows \mathrm{NO}(\mathrm{w})+\mathrm{O}$. For the remaining processes whose statespecific rates are not available, the classical model of Landau-Teller relaxation is employed for the $\mathrm{V}-\mathrm{T}$ data, and the Marrone-Treanor model $^{6}$ is employed for the $\mathrm{D}-\mathrm{R}$ data. The approach adopted herein was detailed in Ref. 61.

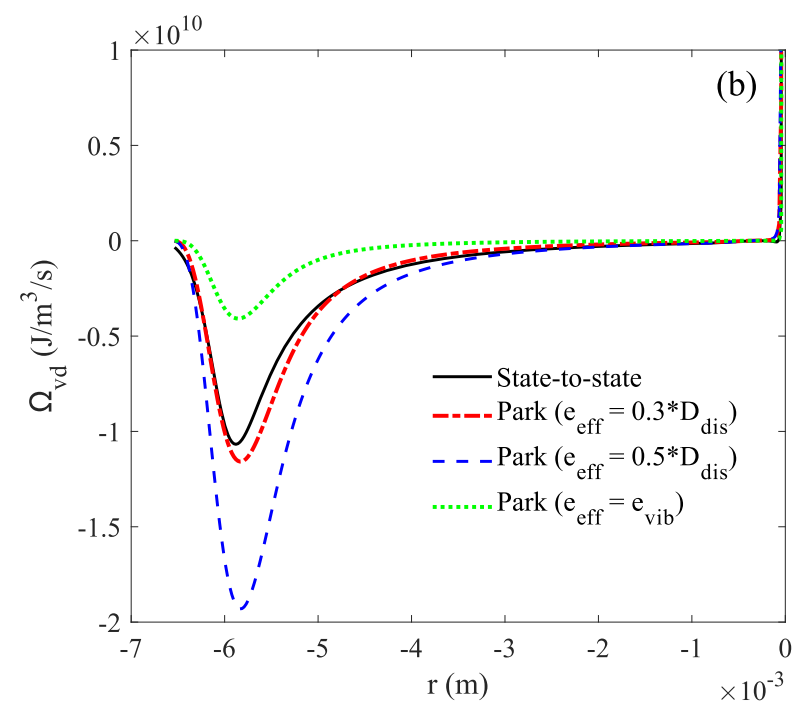

FIG. 8. Nonequilibrium dissociation rates and the vibrational energy losses due to dissociation along the stagnation streamline. (a) Dissociation rates. (b) Vibrational energy losses in dissociation. 
The simulation gives the detailed distributions of macroscopic properties and vibrational levels along the stagnation streamline. Figure 9 shows the profiles of species mole fractions including all vibrational states of $\mathrm{O}_{2}, \mathrm{~N}_{2}$, and NO. It is found that the mole fractions of high vibrational levels of $\mathrm{O}_{2}$ and $\mathrm{N}_{2}$ get rapidly increased behind the shock wave via the $\mathrm{V}-\mathrm{T}$ energy transfer, whereas those of $\mathrm{NO}$ increase slowly because $\mathrm{NO}$ is formed via the Zeldovich exchange reactions after $\mathrm{N}$ and $\mathrm{O}$ are accumulated. Particularly, the profiles of $\mathrm{O}, \mathrm{N}$, and $\mathrm{NO}$ are in a similar trend except that the mole fraction of $\mathrm{O}$ is much larger than that of $\mathrm{N}$; this is because $\mathrm{O}_{2}$ requires lower activation energy for dissociation. Close to the wall surface, the translational temperature is low due to the cold wall (wall temperature is $300 \mathrm{~K}$ ), and then, the vibrational temperature decreases toward to the surface by transferring the vibrational energy to the translational energy. As a result, low-temperature recombination reactions are enhanced near the surface and the formed molecules have very high vibrational energy, which increases the mole fractions of very high level vibrational states.

The state-specific calculation also provides information on the vibrational distributions of molecules along the stagnation streamline. Figures 10 and 11 show the normalized vibrational distributions at typical locations along the stagnation streamline. Behind the shock wave [Fig. 10(a)], the vibrational distributions show nonequilibrium both in species and vibrational levels. For $\mathrm{O}_{2}$ and $\mathrm{N}_{2}$, the vibrational distribution functions feature a large over-population in the high vibrational levels due to multiquanta $\mathrm{V}-\mathrm{T}$ energy transfer. For NO species, the distribution is even complex, which is overpopulation in the middle levels and under-population in high levels. Along the streamline, vibrationally favored dissociation proceeds gradually depend on the value of dissociation energy of specific species. It is found that the high-level $\mathrm{O}_{2}$ molecules get significantly dissociated at $1.5 \mathrm{~mm}$ behind shock front [Fig. 10(b)] and that of $\mathrm{N}_{2}$ occurs at $7.0 \mathrm{~mm}$ [Fig. 10(c)], which results in under-population of high level in the vibrational level distributions of both $\mathrm{O}_{2}$ and $\mathrm{N}_{2}$. Furthermore, the vibrational temperatures of three species get close and nonequilibrium becomes weak as molecules collide along the streamline.

It is also interesting to analyze the behavior of vibrational ladders inside the thermal boundary layer. Figure 11 presents the populations of $\mathrm{O}_{2}, \mathrm{~N}_{2}$, and $\mathrm{NO}$ vibrational levels for three distances away from the wall surface. At $0.4 \mathrm{~mm}$ away from the wall, the vibrational distributions [Fig. 11(a)] are similar to those of Fig. $10(\mathrm{c})$, which means that weak nonequilibrium exists between these locations. At $0.2 \mathrm{~mm}$ away from the wall, the $\mathrm{O}-\mathrm{O}$ recombination leads to the over-population of high vibrational levels of $\mathrm{O}_{2}$ [Fig. 11(b)]. Then, very close to the wall, recombination reactions perturb the vibrational level populations of all molecules appreciably, and large over-population zones are observed in the high level tails. It is also observed that there is a plateau in the distribution functions at the wall surface, which is related to the Treanor distribution.

It should be mentioned that this case has also been simulated using the two-temperature model when the results from the stagnation streamline model is compared with full CFD data in Sec. III. In order to check the difference between the STS and 2T results, the flow temperatures and mass fraction of $\mathrm{O}$ are compared in Fig. 12. It shows that the $2 \mathrm{~T}$ model predicts larger shock standoff distance. The translational temperature predicted by the $2 \mathrm{~T}$ model is also larger at the shock end and inside the boundary layer. The vibrational temperature in the $2 \mathrm{~T}$ results is very close to the vibrational temperature
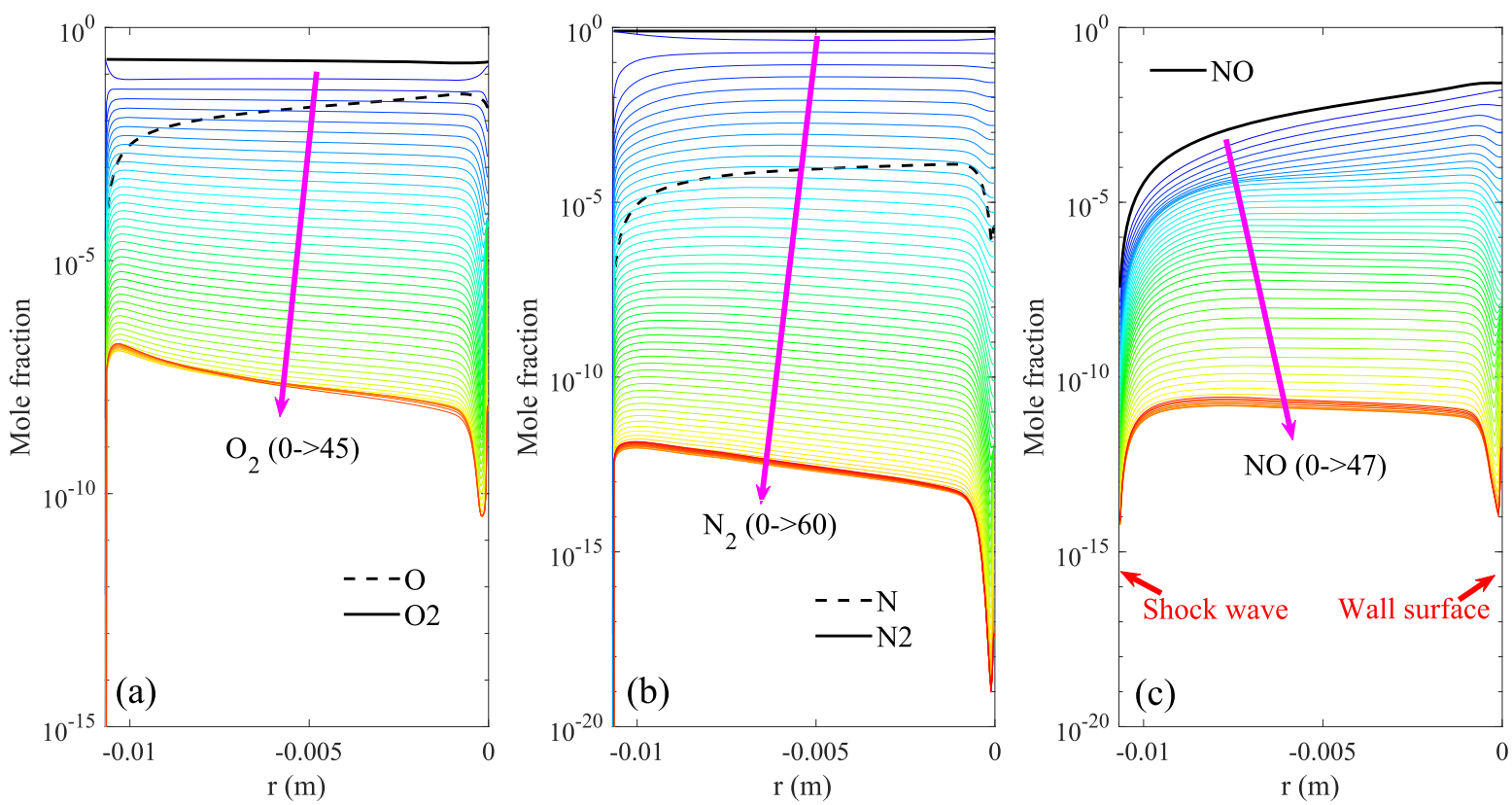

FIG. 9. Species mole fractions along the stagnation streamline. (a) The red line is the total mole fraction of $\mathrm{O}_{2}$, and the black line is the mole fraction of $\mathrm{O}$. (b) The red line is the total mole fraction of $\mathrm{N}_{2}$, and the black line is the mole fraction of $\mathrm{N}$. (c) The black line is the total mole fraction of NO. 

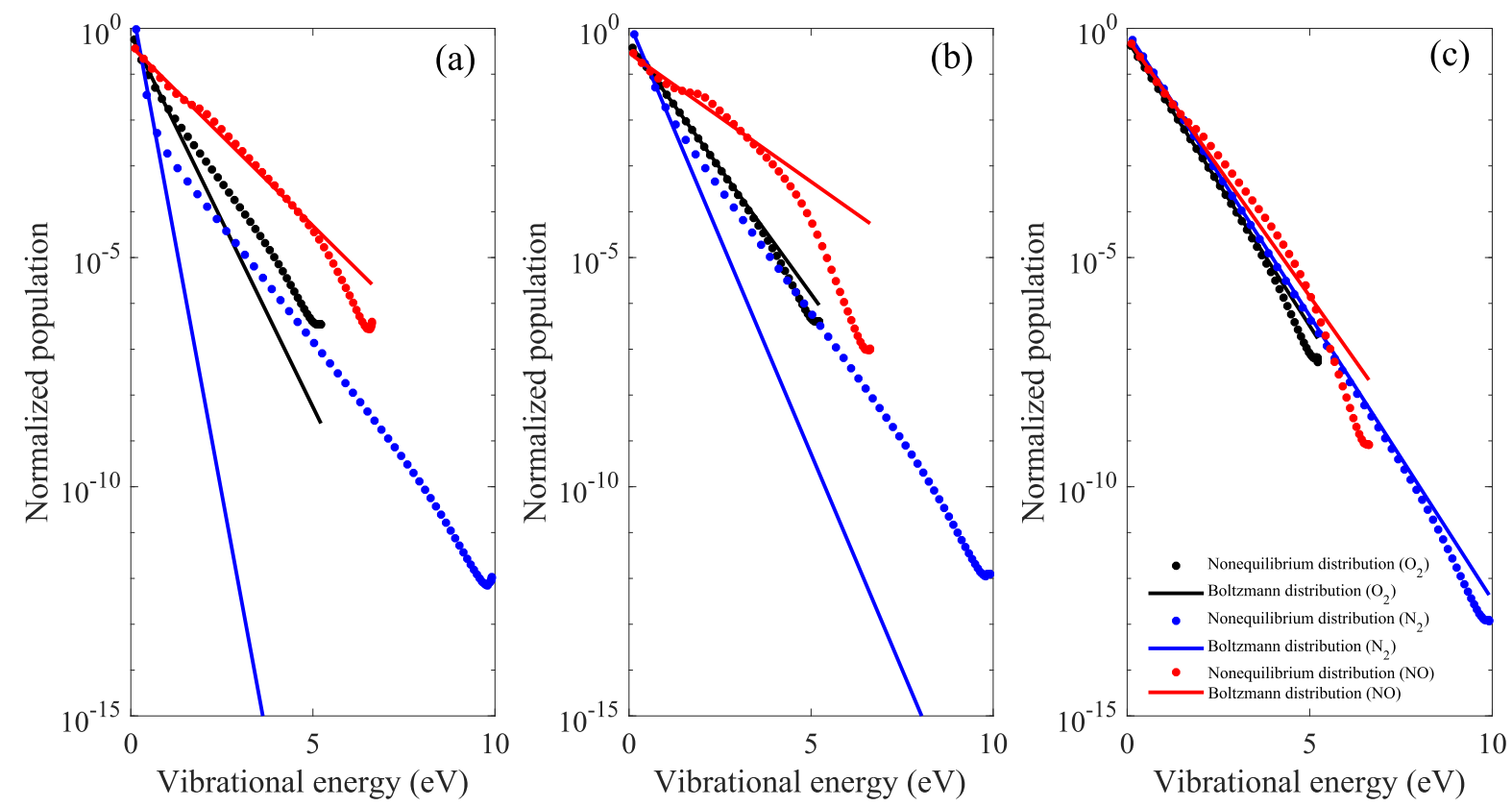

FIG. 10. Vibrational distribution function of molecules at different locations along the stagnation streamline. The symbols are nonequilibrium distribution functions, and the solid lines are Boltzmann distributions under equivalent vibrational temperature. (a) $0.2 \mathrm{~mm}$ behind shock front, (b) $1.5 \mathrm{~mm}$ behind shock front, and (c) $7.0 \mathrm{~mm}$ behind shock front.

of the first excited state [defined in Eq. (24)] of $\mathrm{N}_{2}$ outside of the boundary layer, which is far different from the vibrational temperatures of the first excited state of $\mathrm{O}_{2}$ and $\mathrm{NO}$ in the STS results. In addition, the STS model predicts a much larger dissociation of $\mathrm{O}_{2}$ behind the shock, whereas the $2 \mathrm{~T}$ results show higher recombination of $\mathrm{O}_{2}$ inside the boundary layer. Because of the flow difference between the two models, the heat flux at the wall shows obvious discrepancy with $45.3 \mathrm{~W} / \mathrm{cm}^{2}$ from the STS model and $53.9 \mathrm{~W} / \mathrm{cm}^{2}$
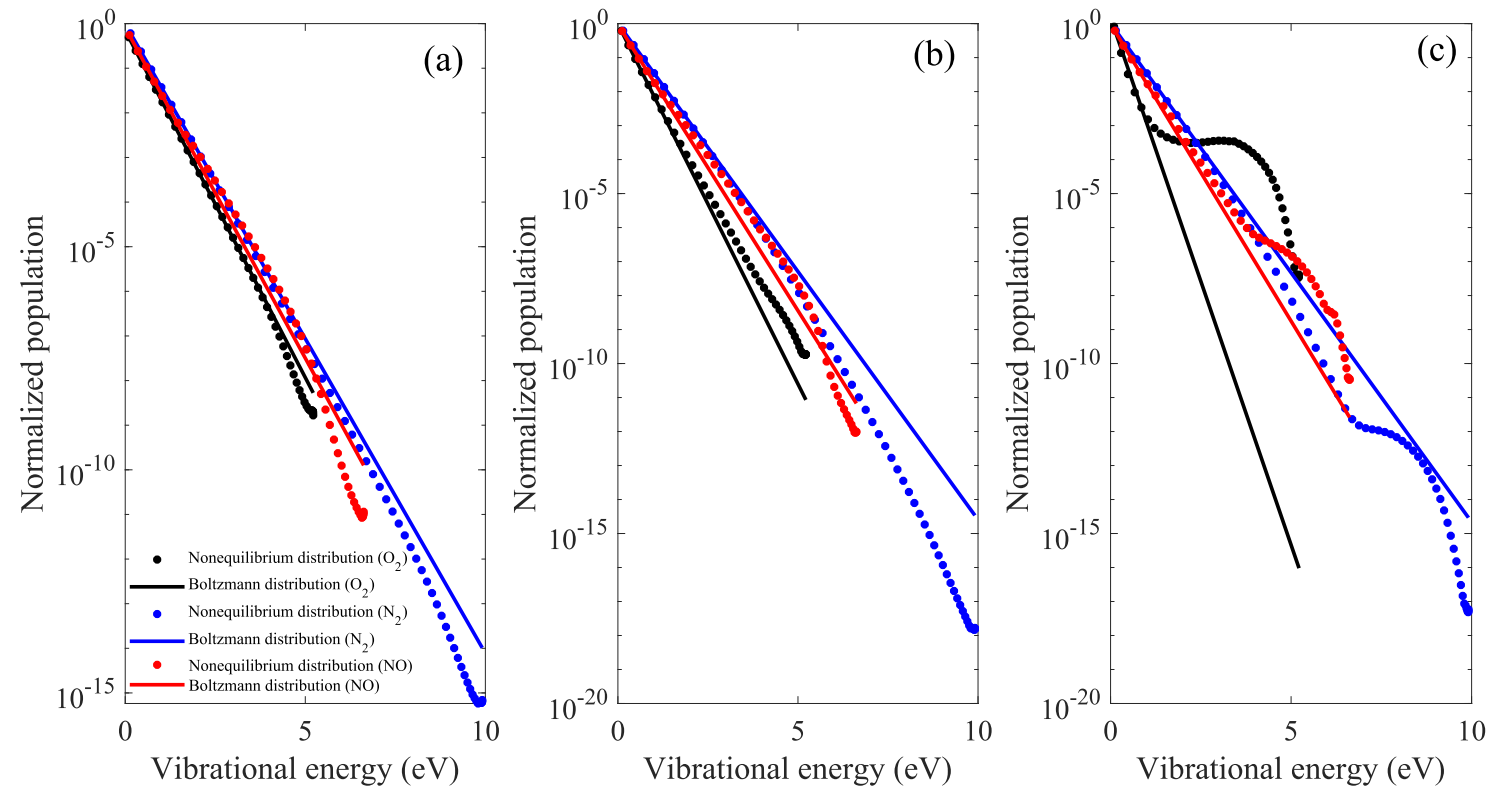

FIG. 11. Vibrational distribution function of molecules at different locations along the stagnation streamline. The symbols are nonequilibrium distribution functions, and the solid lines are Boltzmann distributions under equivalent vibrational temperature. (a) $0.4 \mathrm{~mm}$ away from the wall surface, (b) $0.2 \mathrm{~mm}$ away from the wall surface, and (c) $0.0 \mathrm{~mm}$ away from the wall surface. 

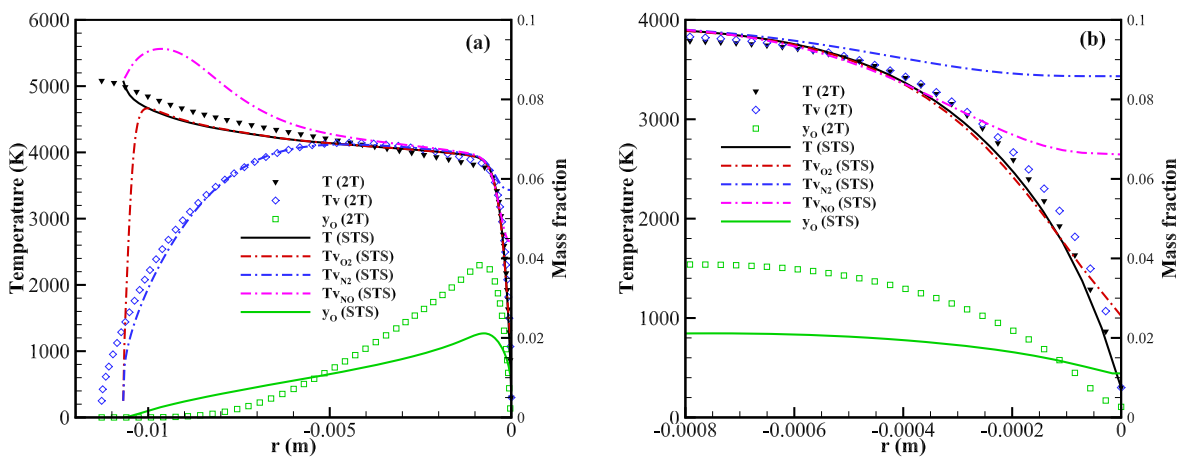

FIG. 12. Comparison of macroscopic flow parameters obtained by the $2 \mathrm{~T}$ and STS models (a) along the stagnation streamline and (b) inside the boundary layer. from the $2 \mathrm{~T}$ model. Given that the compared case is not under a strong nonequilibrium situation (with $M a=10$ and $H=40 \mathrm{~km}$ ), the heat flux might not be correctly predicted using the $2 \mathrm{~T}$ model for nonequilibrium hypersonic flows.

\section{CONCLUSIONS}

In this work, a quasi-one-dimensional stagnation streamline model was proposed to study the thermochemical nonequilibrium flow along the stagnation streamline over a blunt body. The quantum based state-to-state approach and classical multi-temperature models were incorporated into the stagnation streamline model. Detailed comparisons with full field CFD simulations demonstrated that the present model was able to reproduce the thermochemical nonequilibrium flow along the stagnation streamline with very good accuracy. The simulation of $\mathrm{N}_{2} / \mathrm{N} / \mathrm{O}_{2} / \mathrm{O} / \mathrm{NO}$ air mixture with the state-to-state vibrational kinetics was given as an example in this paper. It turns out that the present model is very efficient and can be employed to simulate the state-specific flow characteristics along the stagnation streamline at various flight conditions.

The state-to-state simulation of flow over a blunt body shows that the vibrational energy is in non-Boltzmann distributions both in the vibrational levels and in species. Particularly, the high levels display "over-population" behind the bow shock, and the very high levels display "under-population" at a certain distance away from the shock and "over-population" near the body surface. Therefore, the dissociation rates and vibrational energy losses in dissociation depend on nonequilibrium vibrational distribution, which is very difficult for multi-temperature models to predict. Thus, multitemperature models fail to predict thermochemical processes in hypersonic flows in principle.

The present stagnation streamline model is both efficient and accurate, which is very useful to understand the thermochemical behavior of hypersonic flows. It can generate a large amount of results by simulating flows at various conditions. It can also be employed to adjust the empirical parameters used in available multitemperature models for full field simulations. It is also possible to develop new multi-temperature models by introducing proper nonequilibrium parameters.

\section{ACKNOWLEDGMENTS}

This work was supported by the Strategic Priority Research Program of the Chinese Academy of Sciences (Grant No. XDA17030100) and the National Natural Science Foundation of China (Grant No. 11372325). The authors would like to thank Dr. Jiaao Hao for helpful discussions about the state-to-state approach.

\section{APPENDIX A: TWO-TEMPERATURE MODELS}

The vibrational-translational energy exchange term is usually described by the Landau-Teller equation,

$$
\Omega_{v-t}=\sum_{c} \rho_{c} \frac{e_{c, v i b}\left(T_{t r}\right)-e_{c, v i b}\left(T_{v e}\right)}{\tau_{c}}
$$

where $e_{c, v i b}\left(T_{t r}\right)$ and $e_{c, v i b}\left(T_{v e}\right)$ represent the local equilibrium value and the physical value of the vibrational energy of species $c$, respectively. $\tau_{c}$ is the vibrational-translational relaxation time of species $c$ and expressed by the formula of Millikan and White ${ }^{24}$ with high temperature correction. ${ }^{4}$ In addition, dissociation and recombination reactions lead to the loss and gain of the vibrational energy in the term $\Omega_{v-d}$, which reflects the effect of vibration-dissociation coupling. Actually, the key for dealing with the term $\Omega_{v-d}$ is how to define the vibrational energy loss due to dissociation (i.e., the effective vibrational energy $\left.e_{v i b \text {,eff }}\right)$. Another important point of the vibration-dissociation coupling effect is the nonequilibrium dissociation rate, which is quite different from the equilibrium dissociation rate. Therefore, the nonequilibrium factor was usually introduced to correct the vibration-dissociation coupling effect, ${ }^{53}$

$$
\begin{gathered}
Z\left(T_{t r}, T_{v e}\right)=\frac{k_{f}}{k_{f, e q}}, \\
k_{f, e q}=C T^{n} \exp \left(-\frac{\theta_{d}}{T}\right),
\end{gathered}
$$

where $k$ denotes the reaction rate, subscript $f$ denotes the forward reaction (namely, dissociation reaction), subscript eq denotes the state of equilibrium, and $C, n, \theta_{d}$ are the reaction parameters in the Arrhenius law.

As a widely used two-temperature model, the Park model was proposed in Ref. 5. Park proposed that the dissociation reaction is controlled by the dissociation control temperature $T_{a}=T_{t r}^{q} T_{v e}^{1-q, 5}$, where $q$ is generally taken as 0.5 or 0.7 . The nonequilibrium factor is

$$
Z\left(T_{t r}, T_{v e}\right)=\left(T_{a} T_{t r}^{-1}\right)^{n} \exp \left(\frac{-\theta_{d}}{T_{a}}+\frac{\theta_{d}}{T_{t r}}\right) .
$$

Moreover, Park assumed that the vibrational energy loss due to dissociation is equal to the average vibrational energy of a molecule 
or a constant time dissociation energy of a molecule. ${ }^{5}$ Namely, $e_{v i b, e f f}=\bar{e}_{v i b}$ and $e_{v i b, e f f}=\alpha E_{d}$. Herein, $\bar{e}_{v i b}$ is the average vibrational energy, $E_{d}$ is the dissociation energy, and $\alpha$ is a constant usually set to 0.3. Park's model is found to give reasonable results except for conditions of strong nonequilibrium $\left(T_{t r} \gg T_{v e}\right.$ or $\left.T_{v e} \gg T_{t r}\right)$, where large errors in the dissociation rates are present.

\section{APPENDIX B: GOVERNING EQUATIONS FOR STATE-SPECIFIC QUASI-ONE-DIMENSIONAL SIMULATION}

The quasi-one-dimensional stagnation streamline model coupled with the vibrational state-to-state approach is developed and described in detail in this appendix. The main difference between the state-to-state approach and two-temperature model (as presented in Sec. II B) using the present stagnation streamline model is the species continuity equations. Since each vibrational state is considered as an individual state, the expression of the species continuity equation then becomes

$$
\begin{gathered}
\rho v \frac{\partial y_{c i}}{\partial r}-\frac{\partial}{\partial r}\left(\rho D_{c i} \frac{\partial y_{c i}}{\partial r}\right)-\frac{2 \rho D_{c i}}{r} \frac{\partial y_{c i}}{\partial r}=\dot{\omega}_{c i} \\
c=1, \ldots, n s, i=1, \ldots, \text { levels. }
\end{gathered}
$$

$y_{c i}=\rho_{c i} / \rho$ is the mass fraction of species $c(c=1, \ldots, n s, n s$ is the number of all species $c$ ) at the $i$ th vibrational level, and $D_{c i}$ is the diffusion coefficient of species $c$ at the $i$ th vibrational level (its expression is derived using the modified Chapman-Enskog approximation in Ref. 9). Apart form the species continuity equations, the momentum equations and the conservation equation of total energy are similar between the state-to-state approach and the two-temperature model, as in Eqs. (14)-(16) of Sec. II B. Moreover, the conservation equations of internal energy [i.e., Eq. (17)] are no longer needed in the frame of the state-to-state approach.

As an example, the theoretical details of state-specific quasione-dimensional simulation of binary mixture $\mathrm{O}_{2} / \mathrm{O}$ are provided as follows: Herein, each vibration energy level of $\mathrm{O}_{2}$ is treated as an independent species, and the detailed vibrational distribution is predicted using the master equation. From kinetic theory, we know that each vibrational level of $\mathrm{O}_{2}$ has the opportunity to participate in vibrational transition and dissociation-recombination reactions, which includes vibration-translation $(\mathrm{V}-\mathrm{T})$ energy transfer,

$$
\mathrm{O}_{2}(i)+\mathrm{O} \Leftrightarrow \mathrm{O}_{2}(f)+\mathrm{O}
$$

vibration-vibration-translation $(\mathrm{V}-\mathrm{V}-\mathrm{T})$ energy transfer,

$$
\mathrm{O}_{2}\left(i_{1}\right)+\mathrm{O}_{2}\left(i_{2}\right) \Leftrightarrow \mathrm{O}_{2}\left(f_{1}\right)+\mathrm{O}_{2}\left(f_{2}\right)
$$

and dissociation-recombination reactions,

$$
\mathrm{O}_{2}(i)+M \Leftrightarrow \mathrm{O}+\mathrm{O}+M
$$

Taking into account the above thermochemical processes [Eqs. (B2)-(B4)], one can obtain the production rate of number density for each vibrational level of oxygen as

$$
\begin{aligned}
\frac{\partial\left[\mathrm{O}_{2}(i)\right]}{\partial t}= & \sum_{f \neq i}\left\{k_{V-T}(f \rightarrow i)\left[\mathrm{O}_{2}(f)\right]-k_{V-T}(i \rightarrow f)\left[\mathrm{O}_{2}(i)\right]\right\} \\
& +\sum_{i_{2}} \sum_{f_{1}} \sum_{f_{2}}\left\{\begin{array}{l}
k_{V-V-T}\left(f_{1}, f_{2} \rightarrow i, i_{2}\right)\left[\mathrm{O}_{2}\left(f_{1}\right)\right]\left[\mathrm{O}_{2}\left(f_{2}\right)\right] \\
-k_{V-V-T}\left(i, i_{2} \rightarrow f_{1}, f_{2}\right)\left[\mathrm{O}_{2}(i)\right]\left[\mathrm{O}_{2}\left(i_{2}\right)\right]
\end{array}\right. \\
& +k_{r c c}^{\mathrm{O}_{2}}(i)\left[\mathrm{O}_{2}\right][\mathrm{O}]^{2}-k_{d i s}^{\mathrm{O}_{2}}(i)\left[\mathrm{O}_{2}(i)\right]\left[\mathrm{O}_{2}\right] \\
& +k_{r e c}^{\mathrm{O}}(i)[\mathrm{O}]^{3}-k_{d i s}^{\mathrm{O}}(i)\left[\mathrm{O}_{2}(i)\right][\mathrm{O}],
\end{aligned}
$$

where $k_{V-V-T}$ and $k_{V-T}$ are the state-specific reaction rates of $\mathrm{V}-\mathrm{V}-\mathrm{T}$ and $\mathrm{V}-\mathrm{T}$, respectively, and the forward and backward reaction rates satisfy the microscopic detailed balance principle. $k_{d i s}^{\mathrm{O}_{2}}(i)$ and $k_{\text {rec }}^{\mathrm{O}_{2}}(i)$ are the state-specific dissociation and recombination rates of the vibrational energy level $i$, respectively, [:] represents the number density of a component (the oxygen energy level has been regarded as an independent component), and $m_{\mathrm{O}_{2}}$ is the mass of oxygen molecules. Then, on the basis of the quasi-one-dimensional model, the continuity equation of each vibrational level of $\mathrm{O}_{2}$ is

$$
\begin{gathered}
\rho v \frac{\partial c_{\mathrm{O}_{2}(i)}}{\partial r}-\left(\frac{\partial}{\partial r}+\frac{2}{r}\right)\left(\rho D_{\mathrm{O}_{2}} \frac{\rho_{\mathrm{O}_{2}(i)}}{\rho_{\mathrm{O}_{2}}} \frac{\partial c_{\mathrm{O}_{2}}}{\partial r}\right) \\
-\left(\frac{\partial}{\partial r}+\frac{2}{r}\right)\left[\rho_{\mathrm{O}_{2}} \tilde{D} \frac{\partial}{\partial r}\left(\frac{\rho_{\mathrm{O}_{2}(i)}}{\rho_{\mathrm{O}_{2}}}\right)\right]=\dot{\omega}_{\mathrm{O}_{2}(i)} .
\end{gathered}
$$

In the above expression, it should be noted that in addition to the diffusion between oxygen molecules and oxygen atoms, the diffusion between different vibrational energy levels of oxygen molecules is also included. The diffusion process between $\mathrm{O}_{2}$ and $\mathrm{O}$ is described by the effective binary diffusion, and the effective binary diffusion coefficient is calculated by Gupta's mixing law. ${ }^{62}$ The effective diffusion coefficient between different vibration levels of $\mathrm{O}_{2}$ is ${ }^{63}$

$$
\tilde{\mathrm{D}}=\left(\frac{x_{\mathrm{O}_{2}}}{\vartheta_{\mathrm{O}_{2} \mathrm{O}_{2}}}+\frac{x_{\mathrm{O}}}{\vartheta_{\mathrm{O}_{2} \mathrm{O}}}\right)^{-1},
$$

where $x$ is the molar fraction of the species and $\vartheta$ is the collision integral. atom is

In addition to Eq. (B6), the continuity equation of the oxygen

$$
\rho v \frac{\partial c_{o}}{\partial r}-\frac{\partial}{\partial r}\left(\rho_{\mathrm{O}} D_{o} \frac{\partial c_{o}}{\partial r}\right)-\frac{2}{r} \rho_{o} D_{o} \frac{\partial c_{o}}{\partial r}=\dot{\omega}_{o}
$$

The remaining equations in the quasi-one-dimensional model for the conservation equation of momentum and total energy are the same as discussed in Sec. II B. Again, it should be noted that the conservation equation of vibrational energy is not needed because the number density of each vibrational state has been solved via the continuity equation based on the state-to-state approach.

Furthermore, from the state-to-state approach, the apparent rate constant of $\mathrm{O}_{2}$ dissociation in nonequilibrium state can be defined as

$$
k_{d i s}=\sum_{i} k_{\mathrm{dis}, i} \frac{\left[\mathrm{O}_{2}(i)\right]}{\left[\mathrm{O}_{2}\right]},
$$

where $k_{\mathrm{dis}, i}$ is the dissociation rate constant of $\mathrm{O}_{2}$ at the vibrational level $i$. The added or removed vibrational energy is obtained as 


$$
\Omega_{v, v-d}=\left\{\begin{array}{lc}
\frac{d\left[\mathrm{O}_{2}\right]}{d t} e_{v i b, e f f}, & \text { multi-temperature model } \\
\sum_{i} \frac{d\left[\mathrm{O}_{2}(i)\right]}{d t} e_{i}, & \text { state-to-state approach. }
\end{array}\right.
$$

The results of detailed state-specific quasi-one-dimensional simulation for $\mathrm{O}_{2} / \mathrm{O}$ mixtures are given in Sec. IV A.

\section{REFERENCES}

'P. A. Gnoffo, "Planetary-entry gas dynamics," Annu. Rev. Fluid Mech. 31, 459494 (1999).

${ }^{2}$ G. S. R. Sarma, "Physico-chemical modelling in hypersonic flow simulation," Prog. Aeronaut. Sci. 36, 281-349 (2000).

${ }^{3}$ C. Park, "Hypersonic aerothermodynamics: Past, present and future," Int. J. Aeronaut. Space Sci. 14, 1-10 (2013).

${ }^{4}$ C. Park, Nonequilibrium Hypersonic Aerothermodynamics (Wiley, 1990).

${ }^{5}$ C. Park, "Assessment of two-temperature kinetic model for ionizing air," J. Thermophys. Heat Transfer 3, 233-244 (1989).

${ }^{6} \mathrm{P}$. V. Marrone and C. E. Treanor, "Chemical relaxation with preferential dissociation from excited vibrational levels," Phys. Fluids 6, 1215-1221 (1963).

${ }^{7}$ S. O. Macheret, A. A. Fridman, I. V. Adamovich, J. Rich, and C. E. Treanor, "Mechanisms of nonequilibrium dissociation of diatomic molecules," in 6th Joint Thermophysics and Heat Transfer Conference, Fluid Dynamics and Co-located Conferences (American Institute of Aeronautics and Astronautics, 1994).

${ }^{8}$ E. Kustova, E. Nagnibeda, G. Oblapenko, A. Savelev, and I. Sharafutdinov, "Advanced models for vibrational-chemical coupling in multi-temperature flows," Chem. Phys. 464, 1-13 (2016).

${ }^{9}$ E. Nagnibeda and E. Kustova, Non-Equilibrium Reacting Gas Flows: Kinetic Theory of Transport and Relaxation Processes (Springer Science and Business Media, 2009).

${ }^{10}$ J. G. Kim and I. D. Boyd, "State-resolved master equation analysis of thermochemical nonequilibrium of nitrogen," Chem. Phys. 415, 237-246 (2013).

${ }^{11}$ M. Panesi, A. Munafò, T. Magin, and R. Jaffe, "Nonequilibrium shock-heated nitrogen flows using a rovibrational state-to-state method," Phys. Rev. E 90, 013009 (2014).

${ }^{12}$ I. Armenise and E. V. Kustova, "State-to-state models for $\mathrm{CO}_{2}$ molecules: From the theory to an application to hypersonic boundary layers," Chem. Phys. 415, 269-281 (2013).

${ }^{13}$ G. Shoev, G. Oblapenko, O. Kunova, M. Mekhonoshina, and E. Kustova, "Validation of vibration-dissociation coupling models in hypersonic non-equilibrium separated flows," Acta Astronaut. 144, 147-159 (2018).

${ }^{14} \mathrm{I}$. Armenise and F. Esposito, " $\mathrm{N}_{2}, \mathrm{O}_{2}$, NO state-to-state vibrational kinetics in hypersonic boundary layers: The problem of rescaling rate coefficients to uniform vibrational ladders," Chem. Phys. 446, 30-46 (2015).

${ }^{15}$ E. Kustova, M. Mekhonoshina, and A. Kosareva, "Relaxation processes in carbon dioxide," Phys. Fluids 31, 046104 (2019).

${ }^{16}$ F. Esposito, M. Capitelli, and C. Gorse, "Quasi-classical dynamics and vibrational kinetics of $\mathrm{N}+\mathrm{N}_{2}(v)$ system," Chem. Phys. 257, 193-202 (2000).

${ }^{17} \mathrm{~F}$. Esposito and M. Capitelli, "The relaxation of vibrationally excited $\mathrm{O}_{2}$ molecules by atomic oxygen," Chem. Phys. Lett. 443, 222-226 (2007).

${ }^{18}$ D. A. Andrienko and I. D. Boyd, "State-specific dissociation in $\mathrm{O}_{2}-\mathrm{O}_{2}$ collisions by quasiclassical trajectory method," Chem. Phys. 491, 74-81 (2017).

${ }^{19}$ H. Luo, M. Kulakhmetov, and A. Alexeenko, "Ab initio state-specific $\mathrm{N}_{2}+\mathrm{O}$ dissociation and exchange modeling for molecular simulations," J. Chem. Phys. 146, 074303 (2017).

${ }^{20} \mathrm{M}$. Kulakhmetov, M. Gallis, and A. Alexeenko, "A $b$ initio-informed maximum entropy modeling of rovibrational relaxation and state-specific dissociation with application to the $\mathrm{O}_{2}+\mathrm{O}$ system," J. Chem. Phys. 144, 174302 (2016).

${ }^{21}$ D. A. Andrienko and I. D. Boyd, "Vibrational energy transfer and dissociation in $\mathrm{O}_{2}-\mathrm{N}_{2}$ collisions at hyperthermal temperatures," J. Chem. Phys. 148, 084309 (2018).
${ }^{22}$ M. S. Grover, E. Torres, and T. E. Schwartzentruber, "Direct molecular simulation of internal energy relaxation and dissociation in oxygen," Phys. Fluids 31, 076107 (2019).

${ }^{23}$ L. Landau and E. Teller, "On the theory of sound dispersion," Phys. Z. Sowjetunion 10, 34 (1936).

${ }^{24}$ R. C. Millikan and D. R. White, "Systematics of vibrational relaxation," J. Chem. Phys. 39, 3209-3213 (1963).

${ }^{25}$ R. N. Schwartz, Z. I. Slawsky, and K. F. Herzfeld, "Calculation of vibrational relaxation times in gases," J. Chem. Phys. 20, 1591-1599 (1952).

${ }^{26} \mathrm{E}$. H. Kerner, "Note on the forced and damped oscillator in quantum mechanics," Can. J. Phys. 36, 371-377 (1958).

${ }^{27}$ I. V. Adamovich, S. O. Macheret, J. W. Rich, and C. E. Treanor, "Vibrational relaxation and dissociation behind shock waves. Part 1. Kinetic rate models," AIAA J. 33, 1064-1069 (1995).

${ }^{28}$ I. V. Adamovich, S. O. Macheret, J. W. Rich, and C. E. Treanor, "Vibrational energy transfer rates using a forced harmonic oscillator model," J. Thermophys. Heat Transfer 12, 57-65 (1998).

${ }^{29} \mathrm{O}$. V. Kunova and E. A. Nagnibeda, "State-to-state description of reacting air flows behind shock waves," Chem. Phys. 441, 66-76 (2014).

${ }^{30} \mathrm{~J}$. Hao, J. Wang, and C. Lee, "State-specific simulation of oxygen vibrational excitation and dissociation behind a normal shock," Chem. Phys. Lett. 681, 69-74 (2017).

${ }^{31}$ A. Chikhaoui, E. A. Nagnibeda, E. V. Kustova, and T. Y. Alexandrova, "Modeling of dissociation-recombination in nozzles using strongly non-equilibrium vibrational distributions," Chem. Phys. 263, 111-126 (2001).

${ }^{32}$ I. Armenise and E. V. Kustova, "On different contributions to the heat flux and diffusion in non-equilibrium flows," Chem. Phys. 428, 90-104 (2014).

${ }^{33}$ I. Armenise and M. Capitelli, "State to state vibrational kinetics in the boundary layer of an entering body in earth atmosphere: Particle distributions and chemical kinetics," Plasma Sources Sci. Technol. 14, S9 (2005).

${ }^{34} \mathrm{~A}$. Klomfass and S. Müller, "Calculation of stagnation streamline quantities in hypersonic blunt body flows," Shock Waves 7, 13-23 (1997).

${ }^{35}$ A. Munafò and T. E. Magin, "Modeling of stagnation-line nonequilibrium flows by means of quantum based collisional models," Phys. Fluids 26, 097102 (2014).

${ }^{36} \mathrm{~S}$. Chen and Q. Sun, "A quasi-one-dimensional model for hypersonic reactive flow along the stagnation streamline," Chin. J. Aeronaut. 29, 1517-1526 (2016).

${ }^{37}$ C. Coletti and G. D. Billing, "Vibrational energy transfer in molecular oxygen collisions," Chem. Phys. Lett. 356, 14-22 (2002).

${ }^{38} \mathrm{C}$. Coletti and G. D. Billing, "Rate constants for energy transfer in carbon monoxide," J. Chem. Phys. 113, 4869-4875 (2000).

${ }^{39}$ L. Scalabrin and I. Boyd, "Numerical simulation of weakly ionized hypersonic flow for reentry configurations," in 9th AIAA/ASME Joint Thermophysics and Heat Transfer Conference (American Institute of Aeronautics and Astronautics, 2006), p. 3773.

${ }^{40} \mathrm{H}$. Kaper and J. H. Ferziger, Mathematical Theory of Transport Processes in Gases (North-Holland Publishing, 1972).

${ }^{41} \mathrm{H}$. Olivier, "A theoretical model for the shock stand-off distance in frozen and equilibrium flows," J. Fluid Mech. 413, 345-353 (2000).

${ }^{42} \mathrm{~N}$. Belouaggadia, H. Olivier, and R. Brun, "Numerical and theoretical study of the shock stand-off distance in non-equilibrium flows," J. Fluid Mech. 607, 167-197 (2008).

${ }^{43}$ C.-H. Lee, "Flow field analysis on the stagnation streamline of a blunt body," Int. J. Aeronaut. Space Sci 17, 149-156 (2016).

${ }^{44}$ C.-Y. Wen and H. G. Hornung, "Non-equilibrium dissociating flow over spheres," J. Fluid Mech. 299, 389-405 (1995).

${ }^{45}$ H. G. Hornung, J. Martinez Schramm, and K. Hannemann, "Hypersonic flow over spherically blunted cone capsules for atmospheric entry. Part 1 . The sharp cone and the sphere," J. Fluid Mech. 871, 1097-1116 (2019).

${ }^{46} \mathrm{Z}$. Wang, L. Bao, and B. Tong, "Rarefaction criterion and non-Fourier heat transfer in hypersonic rarefied flows," Phys. Fluids 22, 126103 (2010).

${ }^{47}$ R. N. Gupta, "Viscous shock-layer study of thermochemical nonequilibrium," J. Thermophys. Heat Transfer 10, 257-266 (1996). 
${ }^{48} \mathrm{~T}$. Gokcen, "Effects of freestream nonequilibrium on convective heat transfer to a blunt body," J. Thermophys. Heat Transfer 10, 234-241 (1996).

${ }^{49}$ L. B. Ibraguimova, A. L. Sergievskaya, V. Y. Levashov, O. P. Shatalov, Y. V. Tunik, and I. E. Zabelinskii, "Investigation of oxygen dissociation and vibrational relaxation at temperatures 4000-10 $800 \mathrm{~K}$," J. Chem. Phys. 139, 034317 (2013).

${ }^{50}$ I. Wysong, S. Gimelshein, Y. Bondar, and M. Ivanov, "Comparison of direct simulation Monte Carlo chemistry and vibrational models applied to oxygen shock measurements," Phys. Fluids 26, 043101 (2014).

${ }^{51}$ A. M. Brandis, C. O. Johnston, and B. A. Cruden, "Non-equilibrium radiation for earth entry,” AIAA Paper No. 2016-3690, 2016.

${ }^{52}$ M. L. Da Silva, V. Guerra, and J. Loureiro, "State-resolved dissociation rates for extremely nonequilibrium atmospheric entries," J. Thermophys. Heat Transfer 21, $40-49$ (2007).

${ }^{53} \mathrm{~J}$. Hao, J. Wang, and C. Lee, "Assessment of vibration-dissociation coupling models for hypersonic nonequilibrium simulations," Aerosp. Sci. Technol. 67, 433-442 (2017)

${ }^{54}$ B. Lopez and M. L. Da Silva, "Non-Boltzmann analysis of hypersonic air re-entry flows," in 11th AIAA/ASME Joint Thermophysics and Heat Transfer Conference (American Institute of Aeronautics and Astronautics, 2014), p. 2547.

${ }^{55}$ See http://esther.ist.utl.pt/pages/stellar.html for Stellar database.

${ }^{56} \mathrm{~F}$. Esposito, I. Armenise, and M. Capitelli, "N-N $\mathrm{N}_{2}$ state to state vibrationalrelaxation and dissociation rates based on quasiclassical calculations," Chem. Phys. 331, 1-8 (2006).
${ }^{57}$ F. Esposito, I. Armenise, G. Capitta, and M. Capitelli, "O-O $\mathrm{O}_{2}$ state-to-state vibrational relaxation and dissociation rates based on quasiclassical calculations," Chem. Phys. 351, 91-98 (2008).

${ }^{58}$ M. L. Da Silva, B. Lopez, V. Guerra, and J. Loureiro, "A multiquantum stateto-state model for the fundamental states of air and application to the modeling of high-speed shocked flows," in 5th International Workshop on Radiation of High Temperature Gases in Atmospheric Entry, ESA SP (American Institute of Aeronautics and Astronautics, 2012), Vol. 714.

${ }^{59}$ D. Bose and G. V. Candler, "Thermal rate constants of the $\mathrm{N}_{2}+\mathrm{O} \rightarrow \mathrm{NO}+\mathrm{N}$ reaction using $a b$ initio ${ }^{3} \mathrm{~A}^{\prime \prime}$ and ${ }^{3} \mathrm{~A}^{\prime}$ potential energy surfaces," J. Chem. Phys. 104, 2825-2833 (1996).

${ }^{60}$ D. Bose and G. V. Candler, "Thermal rate constants of the $\mathrm{O}_{2}+\mathrm{N} \rightarrow \mathrm{NO}+\mathrm{O}$ reaction based on the $\mathrm{A}^{2 \prime}$ and $\mathrm{A}^{4 \prime}$ potential-energy surfaces," J. Chem. Phys. 107, 6136-6145 (1997).

${ }^{61}$ W. Su, D. Bruno, and Y. Babou, "State-specific modeling of vibrational relaxation and nitric oxide formation in shock-heated air," J. Thermophys. Heat Transfer 32, 337-352 (2017).

${ }^{62}$ R. N. Gupta, J. M. Yos, R. A. Thompson, and K.-P. Lee, "A review of reaction rates and thermodynamic and transport properties for an 11-species air model for chemical and thermal nonequilibrium calculations to $30000 \mathrm{~K}$," Technical Report NASA Reference Publication No. 1232, National Aeronautics and Space Administration, Washington, DC, 1990.

${ }^{63}$ E. V. Kustova, "On the simplified state-to-state transport coefficients," Chem. Phys. 270, 177-195 (2001). 